\title{
ISOPERIMETRIC INEQUALITIES IN EUCLIDEAN CONVEX BODIES
}

\author{
MANUEL RITORÉ AND EFSTRATIOS VERNADAKIS \\ Dedicated to Carlos Benitez on his 70th birthday
}

\begin{abstract}
In this paper we consider the problem of minimizing the relative perimeter under a volume constraint in the interior of a convex body, i.e., a compact convex set in Euclidean space with interior points. We shall not impose any regularity assumption on the boundary of the convex set. Amongst other results, we shall prove that Hausdorff convergence in the space of convex bodies implies Lipschitz convergence, the continuity of the isoperimetric profile with respect to the Hausdorff distance, and the convergence in Hausdorff distance of sequences of isoperimetric regions and their free boundaries. We shall also describe the behavior of the isoperimetric profile for small volume and the behavior of isoperimetric regions for small volume.
\end{abstract}

\section{INTRODUCTION}

In this work we consider the isoperimetric problem of minimizing perimeter under a given volume constraint inside a convex body, a compact convex set $C \subset \mathbb{R}^{n+1}$ with interior points. The perimeter considered here will be the one relative to the interior of $C$. No regularity assumption on the boundary will be assumed. This problem is often referred to as the partitioning problem.

A way to deal with this problem is to consider the isoperimetric profile $I_{C}$ of $C$, i.e., the function assigning to each $0<v<|C|$ the infimum of the relative perimeter of the sets inside $C$ of volume $v$. The isoperimetric profile can be interpreted as an optimal isoperimetric inequality in $C$. A minimum for this problem will be called an isoperimetric region. The normalized isoperimetric profile $J_{C}$ is defined on the interval $(0,1)$ by $J_{C}(\lambda)=I_{C}(\lambda|C|)$.

The isoperimetric profile of convex bodies with smooth boundary has been intensively considered, and many results are known, such as the concavity of the isoperimetric profile, Sternberg and Zumbrun [53]; the concavity of the $\left(\frac{n+1}{n}\right)$ power of the isoperimetric profile, Kuwert [34]; the connectedness of the reduced boundary of the isoperimetric regions [53]; the behavior of the isoperimetric profile for small volumes, Bérard and Meyer [9]; or the behavior of isoperimetric regions for small volumes, Fall [21. See also [7], 8] and [43. The results in all these papers make strong use of the regularity of the boundary. In particular, in [53] and [34], the $C^{2, \alpha}$ regularity of the boundary implies a strong regularity of the isoperimetric regions

Received by the editors April 26, 2013.

2010 Mathematics Subject Classification. Primary 49Q10, 49Q20, 52B60.

Key words and phrases. Isoperimetric inequalities, isoperimetric profile, convex bodies, polytopes, Hausdorff distance, Lipschitz distance, perimeter-minimizing sets.

Both authors have been supported by MICINN-FEDER grant MTM2010-21206-C02-01 and Junta de Andalucía grants FQM-325 and P09-FQM-5088. 
up to the boundary, except in a singular set of large Hausdorff codimension, which allowed the authors to apply the classical first and second variation formulas for volume and perimeter. The convexity of the boundary then implies the concavity of the profile and the connectedness of the regular part of the free boundary.

To our knowledge, the only known results for non-smooth boundary are the ones by Bokowski and Sperner [11 on isoperimetric inequalities for the Minkowski content in Euclidean convex bodies, the isoperimetric inequality for convex cones by Lions and Pacella 37] using the Brunn-Minkowski inequality, with the characterization of isoperimetric regions by Figalli and Indrei [22, the extension of LevyGromov inequality [27, App. C] to arbitrary convex sets given by Morgan [41, and the extension of the concavity of the $\left(\frac{n+1}{n}\right)$ power of the isoperimetric profile to arbitrary convex bodies by E. Milman [39, §6]. In his work on the isoperimetric profile for small volumes in the boundary of a polytope, Morgan mentions that his techniques can be adapted to handle the case of small volumes in a solid polytope, [40, Remark 3.11], without uniqueness; see Remark after Theorem 3.8 in [40]. We recall that isoperimetric inequalities outside a convex set with smooth boundary have been obtained in [18, [16, [17. Previous estimates on least perimeter in convex bodies have been obtained by Dyer and Frieze [20], Kannan, Lovász and Simonovits 32 and Bobkov [10. In the initial stages of this research the authors were greatly influenced by the paper of Bokowski and Sperner [11; see also [14]. This work is divided into two parts: in the first one the isoperimetric regions in a ball (for the Minkowski content) using spherical symmetrization are characterized; see also [3] and 48. In the second part, given a convex body $C$ so that there is a closed ball $\bar{B}(x, r) \subset C$, a map between $\bar{B}(x, r)$ and $C$ is built, which transforms the volume and the perimeter in a controlled way, allowing to transfer the isoperimetric inequality of the ball to $C$. This map is not bi-Lipschitz, but can be modified to satisfy this property.

In this paper we extend some of the results already known for Euclidean convex bodies with smooth boundary to arbitrary convex bodies, and prove new results for the isoperimetric profile. We begin by considering the Hausdorff and Lipschitz convergences in the space of convex bodies. We prove in Theorem 3.4 that a sequence $C_{i}$ of convex bodies converging to a convex body $C$ in Hausdorff distance also converges in Lipschitz distance. This is done by considering a "natural" sequence of bi-Lipschitz maps $f_{i}: C \rightarrow C_{i}$, defined by (3.6), and proving that $\operatorname{Lip}\left(f_{i}\right), \operatorname{Lip}\left(f_{i}^{-1}\right) \rightarrow 1$. These maps are modifications of the ones used by Bokowski and Sperner in [11] and have the following key property; see Corollary [3.9] If $\bar{B}(0,2 r) \subset C \cap C^{\prime}, C \cup C^{\prime} \subset \bar{B}(0, R)$ and $f: C \rightarrow C^{\prime}$ is the considered map, then $\operatorname{Lip}(f), \operatorname{Lip}\left(f^{-1}\right)$ are bounded above by a constant depending only on $R / r$. This implies (see Theorem 4.11) a uniform non-optimal isoperimetric inequality for all convex bodies with bounded quotient circumradius/inradius. We also prove in Theorem 3.8 that Lipschitz convergence implies convergence in the weak Hausdorff topology (modulo isometries). Let us recall that in Bayle's Ph.D. thesis [7, Thm. 4.2.7] the convergence of the isoperimetric profiles of a sequence of Riemannian manifolds in $\mathcal{M}(n, d, v, \delta)$ converging in Gromov-Hausdorff distance to a Riemannian manifold in the same class was proved. Here $\mathcal{M}(n, d, v, \delta)$ denotes the set of compact $n$-dimensional Riemannian manifolds satisfying $\operatorname{diam}(M, g) \leqslant d$, $\operatorname{vol}(M, g) \geqslant v$, and $\operatorname{Ricci}_{(M, g)} \geqslant(n-1) \delta g$. Let us also recall that the Gromov compactness theorem [27] implies that the space of compact $n$-dimensional Riemannian 
manifolds $(M, g)$ with sectional curvatures satisfying $|K| \leqslant c_{1}, \operatorname{vol}(M, g) \leqslant c_{2}$ and $\operatorname{diam}(M, g) \leqslant c_{3}$ is precompact in the Lipschitz topology; see also [45], 26]. Results proving the convergence of the boundaries of smooth non-compact convex hypersurfaces have been given by Alexander and Ghomi [1].

Using Theorem 3.4 we prove in Theorem 4.1 the pointwise convergence of the normalized isoperimetric profiles. This implies (Corollary 4.2), through approximation by smooth convex bodies, the concavity of the isoperimetric profile $I_{C}$ and of the function $I_{C}^{(n+1) / n}$ for an arbitrary convex body. As observed by Bayle [7, Thm. 2.3.10], the concavity of $I_{C}^{(n+1) / n}$ implies the strict concavity of $I_{C}$. This is an important property that implies the connectedness of an isoperimetric region and its complement (Theorem 4.6). Theorem 4.6 provides an improvement of a result by Sternberg and Zumbrun: we show in Theorem 4.7 that the interface of an isoperimetric region is connected assuming $C^{2, \alpha}$ regularity of $\partial C$. By standard properties of concave functions, we also obtain in Corollary 4.4 the uniform convergence of the normalized isoperimetric profiles $J_{C}$ and of their powers $J_{C}^{(n+1) / n}$ in compact subsets of the interval $(0,1)$. Using the bi-Lipschitz maps constructed in the first section, we show in Theorem 4.12 that a uniform relative isoperimetric inequality, and hence a Poincaré inequality, holds in metric balls of small radius in C.

Using this relative isoperimetric inequality we prove in Theorem 5.5 a key result on the density of an isoperimetric region and its complement, similar to the ones obtained by Leonardi and Rigot [35, which are in fact based on ideas by David and Semmes [19] for quasi-minimizers of the perimeter. Theorem 5.5 is closer to a "clearing out" result as in Massari and Tamanini [38, Thm. 1] (see also 36]) than to a concentration type argument as in Morgan [42, §13.7]. One of the consequences of Theorem [5.5 is a uniform lower density result (Corollary [5.8). The estimates obtained in Theorem 5.5 are stable enough to allow passing to the limit under Hausdorff convergence. Hence we can improve the $L^{1}$ convergence of isoperimetric regions and show in Theorem 5.11 that this convergence is in Hausdorff distance (see [54, $\S 1.3$ ] and [4, Thm. 2.4.5]). We can prove the convergence of the free boundaries in Hausdorff distance in Theorem 5.13 as well. As a consequence, we are able to show in Theorem 5.12 that, given a convex body $C$, for every $0<v<|C|$, there always exists an isoperimetric region with connected free boundary.

Finally, in the last section we consider the isoperimetric profile for small volumes. In the smooth boundary case, Fall [21] showed that for sufficiently small volume, the isoperimetric regions are small perturbations of geodesic spheres centered at a global maximum of the mean curvature, and derived an asymptotic expansion for the isoperimetric profile. We show in Theorem 6.6 that the isoperimetric profile of a convex set for small volumes is asymptotic to the one of its smallest tangent cone, i.e., the one with the smallest solid angle, and that rescaling isoperimetric regions to have volume 1 makes them subconverge in Hausdorff distance to an isoperimetric region in this convex cone, which is a geodesic ball centered at some apex by the recent result of Figalli and Indrei [22. Although in the interior of the convex set we can apply Allard's regularity result for rectifiable varifolds, obtaining high order convergence of the boundaries of isoperimetric sets, we do not dispose of any regularity result at the boundary to ensure convergence up to the boundary (unless both the set and its limit tangent cone have smooth boundary [29]). As a consequence of Theorem [6.6, we show in Theorem 6.8 that the only isoperimetric 
regions of sufficiently small volume inside a convex polytope are geodesic balls centered at the vertices whose tangent cones have the smallest solid angle. The same result holds when the convex set is locally a cone at the points of the boundary with the smallest solid angle. A similar result for the boundary of the polytope was proven by Morgan [40].

We have organized this paper into several sections. In the next one we introduce the basic background and notation. In the third one we shall consider the relation between the Hausdorff and Lipschitz convergence for convex bodies. In the fourth one we shall prove the continuity of the isoperimetric profile with respect to the Hausdorff distance and some consequences; in the fifth one we shall prove the density result and the convergence of isoperimetric regions and their free boundaries in Hausdorff distance. In the last section, we shall study the behavior of the isoperimetric profile and of the isoperimetric regions for small volume.

The results in this paper are intended to be applied to study the behavior of the asymptotic isoperimetric profile of unbounded convex bodies (closed unbounded convex sets with non-empty interior) in Euclidean space.

\section{Preliminaries}

Throughout this paper we shall denote by $C \subset \mathbb{R}^{n+1}$ a compact convex set with non-empty interior. We shall call such a set a convex body. Note that this terminology does not agree with some classical texts such as Schneider [51. As a rule, basic properties of convex sets which are stated without proof in this paper can be easily found in Schneider's monograph.

The Euclidean distance in $\mathbb{R}^{n+1}$ will be denoted by $d$, and the $r$-dimensional Hausdorff measure of a set $E$ by $H^{r}(E)$. The volume of a set $E$ is its $(n+1)$ dimensional Hausdorff measure and we shall denote it by $|E|$. We shall indicate the closure of $E$ by $\operatorname{cl}(E)$ or $\bar{E}$ and the topological boundary by $\partial E$. The open ball of center $x$ and radius $r>0$ will be denoted by $B(x, r)$, and the corresponding closed ball by $\bar{B}(x, r)$.

In the space of convex bodies one may consider two different notions of convergence. Given a convex body $C$ and $r>0$, we define $C_{r}=\left\{p \in \mathbb{R}^{n+1}: d(p, C) \leqslant r\right\}$. The set $C_{r}$ is the tubular neighborhood of radius $r$ of $C$ and is a closed convex set. Given two convex sets $C, C^{\prime}$, we define their Hausdorff distance $\delta\left(C, C^{\prime}\right)$ by

$$
\delta\left(C, C^{\prime}\right)=\inf \left\{r>0: C \subset\left(C^{\prime}\right)_{r}, C^{\prime} \subset C_{r}\right\} .
$$

The space of convex bodies with the Hausdorff distance is a metric space. Bounded sets in this space are relatively compact by Blaschke's Selection Theorem [51, Thm. 1.8.4]. We shall say that a sequence $\left\{C_{i}\right\}_{i \in \mathbb{N}}$ of convex bodies converges to a convex body $C$ in Hausdorff distance if $\lim _{i \rightarrow \infty} \delta\left(C_{i}, C\right)=0$.

Given two convex bodies $C, C^{\prime} \subset \mathbb{R}^{n+1}$, we define its weak Hausdorff distance $\delta_{S}\left(C, C^{\prime}\right)$ by

$$
\delta_{S}\left(C, C^{\prime}\right)=\inf \left\{\delta\left(C, h\left(C^{\prime}\right)\right): h \in \operatorname{Isom}\left(\mathbb{R}^{n+1}\right)\right\} .
$$

The weak Hausdorff distance is non-negative, symmetric, and satisfies the triangle inequality. Moreover, $\delta_{S}\left(C, C^{\prime}\right)=0$ if and only if there exists $h \in \operatorname{Isom}\left(\mathbb{R}^{n+1}\right)$ such that $C=h\left(C^{\prime}\right)$. 
A map $f:(X, d) \rightarrow\left(X^{\prime}, d^{\prime}\right)$ between metric spaces is Lipschitz if there exists a constant $L>0$ so that

$$
d^{\prime}(f(x), f(y)) \leqslant L d(x, y)
$$

for all $x, y \in X$. Sometimes we will refer to such a map as an $L$-Lipschitz map. The smallest constant satisfying (2.3), sometimes called the dilatation of $f$, will be denoted by $\operatorname{Lip}(f)$. A Lipschitz function on $(X, d)$ is a Lipschitz map $f: X \rightarrow \mathbb{R}$, where we consider on $\mathbb{R}$ the Euclidean distance. A map $f: X \rightarrow Y$ is bi-Lipschitz if both $f$ and $f^{-1}$ are Lipschitz maps.

Given two convex bodies $C, C^{\prime}$, we define their Lipschitz distance $d_{L}$ by

$$
d_{L}\left(C, C^{\prime}\right)=\inf _{f \in \operatorname{Lip}\left(C, C^{\prime}\right)}\left\{\log \left(\max \left\{\operatorname{Lip}(f), \operatorname{Lip}\left(f^{-1}\right)\right\}\right)\right\},
$$

where $\operatorname{Lip}\left(C, C^{\prime}\right)$ is the set of bi-Lipschitz maps from $C$ to $C^{\prime}$. We shall say that a sequence $\left\{C_{i}\right\}_{i \in \mathbb{N}}$ of convex bodies converges in Lipschitz distance to a convex body $C$ if $\lim _{i \rightarrow \infty} d_{L}\left(C_{i}, C\right)=0$. The Lipschitz distance is non-negative, symmetric and satisfies the triangle inequality. Moreover, $d_{L}\left(C, C^{\prime}\right)=0$ if and only if $C$ and $C^{\prime}$ are isometric. If a sequence $\left\{C_{i}\right\}_{i \in \mathbb{N}}$ converges to $C$ in the Lipschitz sense, then there is a sequence of bi-Lipschitz maps $f_{i}: C_{i} \rightarrow C$ such that

$$
\lim _{i \rightarrow \infty} \log \left(\max \left\{\operatorname{Lip}\left(f_{i}\right), \operatorname{Lip}\left(f_{i}^{-1}\right)\right\}\right)=0 \text {. }
$$

This implies $\lim _{i \rightarrow \infty} \max \left\{\operatorname{Lip}\left(f_{i}\right), \operatorname{Lip}\left(f_{i}^{-1}\right)\right\}=1$. As $1 \leqslant \operatorname{Lip}\left(f_{i}\right) \operatorname{Lip}\left(f_{i}^{-1}\right)$, we obtain that both $\operatorname{Lip}\left(f_{i}\right), \operatorname{Lip}\left(f_{i}^{-1}\right) \rightarrow 1$. Conversely, if there is a sequence of biLipschitz maps $f_{i}: C_{i} \rightarrow C$ such that $\lim _{i \rightarrow \infty} \operatorname{Lip}\left(f_{i}\right)=\lim _{i \rightarrow \infty} \operatorname{Lip}\left(f_{i}^{-1}\right)=1$, then $\lim _{i \rightarrow \infty} d_{L}\left(C_{i}, C\right)=0$.

If $M, N$ are subsets of Euclidean spaces and $f: M \rightarrow N$ is a Lipschitz map, then $g: \lambda M \rightarrow \lambda N$ defined by $g(x)=\lambda f\left(\frac{x}{\lambda}\right), x \in \lambda M, \lambda>0$, is a Lipschitz map so that $\operatorname{Lip}(g)=\operatorname{Lip}(f)$. This yields the very useful consequence

$$
d_{L}(\lambda M, \lambda N)=d_{L}(M, N), \quad \lambda>0 .
$$

For future reference, we list the following properties of Lipschitz maps and functions.

\section{Lemma 2.1.}

(i) Let $f$ be a Lipschitz function on $(X, d)$ so that $|f| \geqslant M>0$. Then $1 / f$ is a Lipschitz function and $\operatorname{Lip}(1 / f) \leqslant \operatorname{Lip}(f) / M^{2}$.

(ii) Let $f_{1}, f_{2}$ be Lipschitz functions on $(X, d)$. Then $f_{1}+f_{2}$ is a Lipschitz function and $\operatorname{Lip}\left(f_{1}+f_{2}\right) \leqslant \operatorname{Lip}\left(f_{1}\right)+\operatorname{Lip}\left(f_{2}\right)$.

(iii) Let $f_{1}, f_{2}$ be Lipschitz functions on $(X, d)$ so that $\left|f_{i}\right| \leqslant M_{i}, i=1,2$. Then $f_{1} f_{2}$ is a Lipschitz function and $\operatorname{Lip}\left(f_{1} f_{2}\right) \leqslant M_{1} \operatorname{Lip}\left(f_{2}\right)+M_{2} \operatorname{Lip}\left(f_{1}\right)$.

(iv) If $\lambda:(X, d) \rightarrow \mathbb{R}$ is Lipschitz with $|\lambda| \leqslant L^{\prime}$, and $f:(X, d) \rightarrow \mathbb{R}^{n}$ is Lipschitz with $|f|<M^{\prime}$, then $\operatorname{Lip}(\lambda f) \leqslant M^{\prime} \operatorname{Lip}(\lambda)+L^{\prime} \operatorname{Lip}(f)$.

(v) If $f_{i}$ are Lipschitz maps that converge pointwise to a Lipschitz map $f$, then $\operatorname{Lip}(f) \leqslant \liminf _{i \rightarrow \infty} \operatorname{Lip}\left(f_{i}\right)$.

The behavior of the Hausdorff measure [13, $§$ 1.7.2] with respect to Lipschitz maps is well known.

Lemma 2.2. Let $C, C^{\prime} \subset \mathbb{R}^{n+1}$ and $f: C \rightarrow C^{\prime}$ be a Lipschitz map. Then, for every $s>0$ and $E \subset C$ we have

$$
H^{s}(f(E)) \leqslant \operatorname{Lip}(f)^{s} H^{s}(E) .
$$


Morever, if $f$ is bi-Lipschitz, then we have

$$
\frac{1}{\operatorname{Lip}\left(f^{-1}\right)^{s}} H^{s}(E) \leqslant H^{s}(f(E)) \leqslant \operatorname{Lip}(f)^{s} H^{s}(E) .
$$

For $t \geqslant 0$, let $E(t)$ denote the set of points of density $t$ of $E$ in $C$ :

$$
E(t)=\left\{x \in C: \lim _{r \rightarrow 0} \frac{\left|E \cap B_{C}(x, r)\right|}{\left|B_{C}(x, r)\right|}=t\right\} .
$$

Since $|E \cap \partial C|=0$, we have that $|E(t)|=|E(t) \cap \operatorname{int}(C)|$. By the LebesgueBesicovitch Theorem we have $|E(1)|=|E|$ and similarly $|E(0)|=|C \backslash E|$.

For $E \subset C$, we define the perimeter of $E$ in the interior of $C$ by

$$
P_{C}(E)=P(E, \operatorname{int}(C))=\sup \left\{\int_{E} \operatorname{div} \xi d H^{n+1}, \xi \in \mathfrak{X}_{0}(\operatorname{int}(C)),|\xi| \leqslant 1\right\},
$$

where $\mathfrak{X}_{0}(\operatorname{int}(C))$ is the set of smooth vector fields with compact support in the interior of $C$. We shall say that $E$ has finite perimeter in $\operatorname{int}(C)$ if $P_{C}(E)<$ $\infty$. A set $E$ of finite perimeter in $\operatorname{int}(C)$ satisfies $P(E) \leqslant P_{C}(E)+H^{n}(\partial C)$ and so is a Caccioppoli set in $\mathbb{R}^{n+1}$. We can define its reduced boundary $\partial^{*} E$ as in [24, Chapter 3] and we have $P_{C}(E)=H^{n}\left(\partial^{*} E \cap \operatorname{int}(C)\right)$.

We define the isoperimetric profile of $C$ by

$$
I_{C}(v)=\inf \left\{P_{C}(E): E \subset C,|E|=v\right\} .
$$

We shall say that $E \subset C$ is an isoperimetric region if $P_{C}(E)=I_{C}(|E|)$. The renormalized isoperimetric profile of $C$ is

$$
Y_{C}=I_{C}^{(n+1) / n} \text {. }
$$

We shall denote by $J_{C}:[0,1] \rightarrow \mathbb{R}^{+}$the normalized isoperimetric profile function

$$
J_{C}(\lambda)=I_{C}(\lambda|C|) .
$$

We shall also denote by $y_{C}:[0,1] \rightarrow \mathbb{R}^{+}$the function

$$
y_{C}=J_{C}^{(n+1) / n} \text {. }
$$

Standard results of Geometric Measure Theory imply that isoperimetric regions exist in a convex body. The following basic properties are well known.

Lemma 2.3. Let $C \subset \mathbb{R}^{n+1}$ be a convex body. Consider a sequence $\left\{E_{i}\right\}_{i \in \mathbb{N}} \subset C$ of subsets with finite perimeter in the interior of $C$.

(i) If $E_{i}$ converges to a set $E \subset C$ with finite perimeter in $\operatorname{int}(C)$ in the $L^{1}(\operatorname{int}(C))$ sense, then $P_{C}(E) \leqslant \liminf _{i \rightarrow \infty} P_{C}\left(E_{i}\right)$.

(ii) If $P_{C}\left(E_{i}\right)$ is uniformly bounded from above, then there exists a set $E \subset C$ of finite perimeter in int $(C)$ such that a subsequence of $\left\{E_{i}\right\}_{i \in \mathbb{N}}$ converges to $E$ in the $L^{1}(\operatorname{int}(C))$ sense.

(iii) Isoperimetric regions exist in $C$ for every volume.

(iv) $I_{C}$ is continuous.

Proof. Properties (i), (ii) and (iii) follow from the lower semicontinuity of perimeter [24, Thm. 1.9] and compactness [24, Thm. 1.19]. The continuity of the isoperimetric profile was proven in [23, Lemma 6.2]. 
For a convex body $C$, the continuity of the isoperimetry profile of $C$ will be a trivial consequence of the concavity of $I_{C}$ proven in Corollary 4.2.

The known results on the regularity of isoperimetric regions are summarized in the following lemma.

Lemma 2.4 ([25, 28, [53, Thm. 2.1]). Let $C \subset \mathbb{R}^{n+1}$ be a convex body and $E \subset C$ an isoperimetric region. Then $\partial E \cap \operatorname{int}(C)=S_{0} \cup S$, where $S_{0} \cap S=\emptyset$ and

(i) $S$ is an embedded $C^{\infty}$ hypersurface of constant mean curvature,

(ii) $S_{0}$ is closed and $H^{s}\left(S_{0}\right)=0$ for any $s>n-7$.

Moreover, if the boundary of $C$ is of class $C^{2, \alpha}$, then $\operatorname{cl}(\partial E \cap \operatorname{int}(C))=S \cup S_{0}$, where

(iii) $S$ is an embedded $C^{2, \alpha}$ hypersurface of constant mean curvature,

(iv) $S_{0}$ is closed and $H^{s}\left(S_{0}\right)=0$ for any $s>n-7$,

(v) at points of $S \cap \partial C, S$ meets $\partial C$ orthogonally.

\section{HAusdorfF AND LipsChitz CONVERGENCE \\ IN THE SPACE OF CONVEX BODIES}

As a first step in our study of the isoperimetric profile of a convex body, we need to prove that Hausdorff convergence of convex bodies implies Lipschitz convergence. We shall also prove the converse replacing the Hausdorff distance by the weak Hausdorff distance as defined in (2.2). We need first some preliminary results for convex sets.

Given a convex body $C \subset \mathbb{R}^{n}$ containing 0 in its interior, its radial function $\rho(C, \cdot): \mathbb{S}^{n} \rightarrow \mathbb{R}$ is defined by

$$
\rho(C, u)=\max \{\lambda \geqslant 0: \lambda u \in C\} .
$$

From this definition it follows that $\rho(C, u) u \in \partial C$ for all $u \in \mathbb{S}^{n}$.

Lemma 3.1. Let $C \subset \mathbb{R}^{n+1}$ be a convex body so that $B(0, r) \subset C \subset B(0, R)$. Then the radial function $\rho(C, \cdot): \mathbb{S}^{n} \rightarrow \mathbb{R}$ is $R^{2} / r$-Lipschitz.

Proof. Let $C^{*}$ be the polar body of $C$ [51, $\left.\S 1.6\right]$. Theorem 1.6.1 in [51] implies that $\left(C^{*}\right)^{*}=C$ and that $B(0,1 / R) \subset C^{*} \subset B(0,1 / r)$. Let $h\left(C^{*}, \cdot\right)$ be the support function of $C^{*}$. Using $\left(C^{*}\right)^{*}=C$, Remark 1.7.7 in 51 implies

$$
\rho(C, u)=\frac{1}{h\left(C^{*}, u\right)} .
$$

By Lemma 1.8.10 in [51] the function $h\left(C^{*}, \cdot\right)$ is $1 / r$-Lipschitz. Since $h\left(C^{*}, \cdot\right) \geqslant$ $1 / R$, we conclude from Lemma 2.1 that $\rho(C, \cdot)$ is an $R^{2} / r$-Lipschitz function.

Lemma 3.2. Let $\left\{C_{i}\right\}_{i \in \mathbb{N}}$ be a sequence of convex bodies converging in Hausdorff distance to a convex body $C$. We further assume that there exist $r, R>0$ such that $B(0, r) \subset \operatorname{int}\left(C_{i}\right) \subset B(0, R)$ for all $i \in \mathbb{N}$, and $B(0, r) \subset \operatorname{int}(C) \subset B(0, R)$. Then

$$
\lim _{i \rightarrow \infty} \sup _{u \in \mathbb{S}^{n}}\left|\rho\left(C_{i}, u\right)-\rho(C, u)\right|=0 .
$$

Proof. We reason by contradiction. Assume there exists $\varepsilon>0$ and $u_{i} \in \mathbb{S}^{n}$ so that a subsequence satisfies

$$
\left|\rho\left(C_{i}, u_{i}\right)-\rho\left(C, u_{i}\right)\right| \geqslant \varepsilon
$$

Passing again to a subsequence we may assume that $u_{i} \rightarrow u \in \mathbb{S}^{n}$. We define

$$
x_{i}=\rho\left(C_{i}, u_{i}\right) u_{i} \in \partial C_{i}, \quad y_{i}=\rho\left(C, u_{i}\right) u_{i} \in \partial C .
$$


Since $\rho\left(C_{i}, \cdot\right)$ and $\rho(C, \cdot)$ are uniformly bounded, we may extract again convergent subsequences $x_{i} \rightarrow x$ and $y_{i} \rightarrow y$. Since $\partial C$ is closed, we have $y \in \partial C$. Since $C_{i} \rightarrow C$ in Hausdorff distance, we have $x \in \partial C$ (it is straightforward to check that $x \notin \mathbb{R}^{n+1} \backslash C$ and that $x \notin \operatorname{int}(C)$ by Lemma 1.8.14 in [51]). Since $\left|x_{i}-y_{i}\right| \geqslant \varepsilon$ we get $|x-y| \geqslant \varepsilon$, but both $x, y$ belong to the ray emanating from 0 with direction $u$. This is a contradiction since $0 \in \operatorname{int}(C)$ [51, Lemma 1.1.8].

Lemma 3.3. Let $\left\{f_{i}\right\}_{i \in \mathbb{N}}$ be a sequence of convex functions defined on a convex open set $C$ and converging uniformly on $C$ to a convex function $f$.

(i) Let $\left\{x_{i}\right\}_{i \in \mathbb{N}}$ be a sequence such that $x=\lim _{i \rightarrow \infty} x_{i}$. If $\nabla f_{i}\left(x_{i}\right), \nabla f(x)$ exist for all $i \in \mathbb{N}$, then $\nabla f_{i}\left(x_{i}\right) \rightarrow \nabla f(x)$.

(ii) $\operatorname{Lip}\left(f_{i}-f\right) \rightarrow 0$.

(iii) If $g$ is a convex function defined in a convex body $C$, then

$$
\operatorname{Lip}(g)=\sup _{z \in D}|\nabla g(z)|,
$$

where $D$ is the subset of $C$ (dense and of full measure) where $\nabla g$ exists.

Proof. The proof of (i) is taken from [49, Thm. 25.7]. We give it for completeness. Assume that $\nabla f_{i}\left(x_{i}\right)$ does not converge to $\nabla f(x)$. Then there exists $y \in \mathbb{R}^{n}$ and $\varepsilon>0$ such that either

$$
\begin{aligned}
& \left\langle\nabla f_{i}\left(x_{i}\right), y\right\rangle-\langle\nabla f(x), y\rangle \geqslant \varepsilon, \quad \text { or } \\
& \left\langle\nabla f_{i}\left(x_{i}\right), y\right\rangle-\langle\nabla f(x), y\rangle \leqslant-\varepsilon
\end{aligned}
$$

holds for a subsequence.

Let us assume that the second inequality in (3.1) holds for a subsequence. For simplicity, we assume it holds for the whole sequence. Thus we have $\left\langle\nabla f_{i}\left(x_{i}\right), y\right\rangle \leqslant$ $\langle\nabla f(x), y\rangle-\varepsilon$ for any index $i$. Multiplying this inequality by $t<0$ we obtain $\left\langle\nabla f_{i}\left(x_{i}\right), t y\right\rangle \geqslant(\langle\nabla f(x), y\rangle-\varepsilon) t$. From this inequality and the convexity of $f_{i}$ we get

$$
f_{i}\left(x_{i}+t y\right)-f_{i}\left(x_{i}\right) \geqslant\left\langle\nabla f_{i}\left(x_{i}\right), t y\right\rangle \geqslant(\langle f(x), y\rangle-\varepsilon) t .
$$

Letting $i \rightarrow \infty$, taking into account that $f_{i} \rightarrow f$ uniformly, we find

$$
\frac{f(x+t y)-f(x)}{t} \leqslant\langle\nabla f(x), y\rangle-\varepsilon .
$$

Taking limits when $t \uparrow 0$ we get $\langle\nabla f(x), y\rangle \leqslant\langle\nabla f(x), y\rangle-\varepsilon$, and we reach a contradiction. The case of the first inequality in (3.1) is treated in the same way. This proves (i).

To prove (ii) we also reason by contradiction, so we assume there exists $\varepsilon>0$ so that $\operatorname{Lip}\left(f_{i}-f\right)>\varepsilon$ holds for a subsequence. For simplicity, we assume that every index $i$ satisfies this inequality. We can find sequences $\left\{x_{i}\right\}_{i \in \mathbb{N}},\left\{y_{i}\right\}_{i \in \mathbb{N}}$ such that $x_{i} \neq y_{i}$ and

$$
\left|\left(f_{i}-f\right)\left(x_{i}\right)-\left(f_{i}-f\right)\left(y_{i}\right)\right|>\varepsilon\left|x_{i}-y_{i}\right| \quad \text { for all } i \in \mathbb{N} .
$$

Passing again to a subsequence if necessary, we assume that there are points $x, y$ such that $x=\lim _{i \rightarrow \infty} x_{i}, y=\lim _{i \rightarrow \infty} y_{i}$.

We observe that it can be assumed that both $\nabla f_{i}$ and $\nabla f$ are defined $H^{1}$-almost everywhere in the segment $\left[x_{i}, y_{i}\right]$ : otherwise we consider a right circular cylinder $D \times\left[x_{i}, y_{i}\right]$ of axis $\left[x_{i}, y_{i}\right]$ so that in every segment parallel to $\left[x_{i}, y_{i}\right]$ of height $\left|x_{i}-y_{i}\right|$, inequality (3.2) is satisfied by its extreme points. Since the set where the gradients $\nabla f_{i}, \nabla f$ exist has full $H^{n+1}$-measure in $D \times\left[x_{i}, y_{i}\right]$ [49, Thm. 25.4], Fubini's 
Theorem implies that for $H^{n}$-almost everywhere in $D$, the gradients are $H^{1}$-almost everywhere defined. We replace $\left[x_{i}, y_{i}\right]$ by one of such segments if necessary.

For $\lambda \in[0,1]$ and $i \in \mathbb{N}$, we define convex functions $u_{i}, v_{i}$ by

$$
u_{i}(\lambda):=\frac{f_{i}\left(x_{i}+\lambda\left(y_{i}-x_{i}\right)\right)-f_{i}\left(x_{i}\right)}{\left|y_{i}-x_{i}\right|}, \quad v_{i}(\lambda):=\frac{f\left(x_{i}+\lambda\left(y_{i}-x_{i}\right)\right)-f\left(x_{i}\right)}{\left|y_{i}-x_{i}\right|} .
$$

Hence (3.2) is equivalent to

$$
\lim _{i \rightarrow \infty}\left(u_{i}(1)-v_{i}(1)\right) \geqslant \varepsilon .
$$

We easily find

$$
\left(u_{i}(\lambda)-v_{i}(\lambda)\right)^{\prime}=f_{i}{ }^{\prime}\left(x_{i}+\lambda\left(y_{i}-x_{i}\right) ; \frac{x_{i}-y_{i}}{\left|x_{i}-y_{i}\right|}\right)-f^{\prime}\left(x_{i}+\lambda\left(y_{i}-x_{i}\right) ; \frac{x_{i}-y_{i}}{\left|x_{i}-y_{i}\right|}\right),
$$

where the derivative $f^{\prime}(p ; u)$ of the convex function $f$ at the point $p$ in the direction of $u$ is defined as in [49, p. 213]. At the points where both $\nabla f_{i}, \nabla f$ exist we get

$$
\left(u_{i}(\lambda)-v_{i}(\lambda)\right)^{\prime}=\left\langle\left(\nabla f_{i}-\nabla f\right)\left(x_{i}+\lambda\left(y_{i}-x_{i}\right), \frac{x_{i}-y_{i}}{\left|x_{i}-y_{i}\right|}\right)\right\rangle
$$

and

$$
\left|\left(u_{i}(\lambda)-v_{i}(\lambda)\right)^{\prime}\right| \leqslant\left|\nabla f_{i}\left(x_{i}+\lambda\left(y_{i}-x_{i}\right)\right)-\nabla f\left(x_{i}+\lambda\left(y_{i}-x_{i}\right)\right)\right| .
$$

By (i) and [49, Thm. 25.5] we have $\lim _{i \rightarrow \infty}\left(u_{i}(\lambda)-v_{i}(\lambda)\right)^{\prime}=0$. By [49, Thm. 10.6], $\operatorname{Lip}\left(f_{i}\right)$ is uniformly bounded. So $\left(u_{i}-v_{i}\right)^{\prime}$ is bounded by a constant by (iii). Then by the Dominated Convergence Theorem [49, Corollary 24.2.1] and the fact that $u_{i}(0)=v_{i}(0)=0$, we get

$$
\lim _{i \rightarrow \infty}\left(u_{i}(1)-v_{i}(1)\right)=\lim _{i \rightarrow \infty} \int_{0}^{1}\left(u_{i}(\lambda)-v_{i}(\lambda)\right)^{\prime} d \lambda=0,
$$

which, together with (3.4), gives a contradiction. Hence $\lim _{i \rightarrow \infty} \operatorname{Lip}\left(f_{i}-f\right)=0$.

To prove (iii), let $z \in D$. There is $w \in \mathbb{S}^{n}$ such that $|\nabla g(z)|=\langle\nabla g(z), w\rangle$. Hence

$$
|\nabla g(z)|=\left|\lim _{\lambda \rightarrow 0} \frac{g(z+\lambda w)-g(z)}{\lambda}\right| \leqslant \sup _{x \neq y} \frac{|g(x)-g(y)|}{|x-y|}=\operatorname{Lip}(g) .
$$

To prove the reverse inequality, take $x, y \in C$ and assume for the moment that $\nabla g$ exists $H^{1}$-almost everywhere in the segment $[x, y]$. Then by [49, Corollary 24.2.1] we have

$$
|g(x)-g(y)|=\left|\int_{0}^{1}\langle\nabla g(x+\lambda(y-x), y-x)\rangle d \lambda\right| \leqslant \sup _{z \in D}|\nabla g(z)||x-y| .
$$

If $\nabla g$ does not exist $H^{1}$-almost everywhere in the segment $[x, y]$, we can make an approximation argument, as in the proof of (ii), with segments parallel to $[x, y]$, where $\nabla g$ exists $H^{1}$ - almost everywhere, to conclude the proof.

Now we prove that Hausdorff convergence of a sequence of convex bodies implies Lipschitz convergence.

Theorem 3.4. Let $\left\{C_{i}\right\}_{i \in \mathbb{N}}$ be a sequence of convex bodies in $\mathbb{R}^{n+1}$ that converges in Hausdorff distance to a convex body $C$. Then $\left\{C_{i}\right\}_{i \in \mathbb{N}}$ converges to $C$ in Lipschitz distance. 
Proof. Translating the whole sequence and its limit we assume that $0 \in \operatorname{int}(C)$. Let $r>0$ so that $\bar{B}(0,2 r) \subset \operatorname{int}(C)$. By [51, Lemma 1.8.14] and the convergence of $C_{i}$ to $C$ in Hausdorff distance, there exists $i_{0} \in \mathbb{N}$ such that $\bar{B}(0, r) \subset \operatorname{int}\left(C_{i}\right)$ for $i \geqslant i_{0}$. Let us denote by $\rho_{i}$ and $\rho$ the radial functions $\rho\left(C_{i}, \cdot\right)$ and $\rho(C, \cdot)$, respectively. Since the sequence $\left\{C_{i}\right\}_{i \in \mathbb{N}}$ converges to $C$ in Hausdorff distance, there exists $R>0$ so that $\bigcup_{i \in \mathbb{N}} C_{i} \cup C \subset B(0, R)$.

For $i \geqslant i_{0}$, we define a map $f_{i}: C \rightarrow C_{i}$ by

$$
f_{i}(x)= \begin{cases}x, & |x| \leqslant r, \\ r \frac{x}{|x|}+(|x|-r) \frac{\rho_{i}\left(\frac{x}{|x|}\right)-r}{\rho\left(\frac{x}{|x|}\right)-r} \frac{x}{|x|}, & |x| \geqslant r .\end{cases}
$$

Using Lemmata 2.1 and 3.1 we obtain that $f_{i}$ is a Lipschitz function. The inverse mapping can be defined exchanging the roles of $\rho_{i}$ and $\rho$ to conclude that $f_{i}$ is a bi-Lipschitz map. The function $f_{i}$ can be rewritten as

$$
f_{i}(x)=x+\left(1-\frac{\rho_{i}\left(\frac{x}{|x|}\right)-r}{\rho\left(\frac{x}{|x|}\right)-r}\right)(r-|x|) \frac{x}{|x|}, \quad|x| \geqslant r .
$$

To show that the sequence $\left\{C_{i}\right\}_{i \in \mathbb{N}}$ converges in Lipschitz distance to $C$, it is enough to prove that both $\operatorname{Lip}\left(f_{i}\right), \operatorname{Lip}\left(f_{i}^{-1}\right)$ converge to 1 . We shall show

$$
\lim _{i \rightarrow \infty} \operatorname{Lip}\left(1-\frac{\rho_{i}\left(\frac{x}{|x|}\right)-r}{\rho\left(\frac{x}{|x|}\right)-r}\right)=0,
$$

and the corresponding inequality interchanging $\rho_{i}$ and $\rho$. From (3.8) and the expression of $f_{i}$ given by (3.7) we would get $\lim \sup _{i \rightarrow \infty} \operatorname{Lip}\left(f_{i}\right) \leqslant 1$. Since $\operatorname{Lip}\left(f_{i}\right) \geqslant$ $\operatorname{Lip}\left(\left.f_{i}\right|_{\bar{B}(0, r)}\right)=1$ we obtain $1 \leqslant \liminf _{i \rightarrow \infty} \operatorname{Lip}\left(f_{i}\right)$. Crossing both inequalities we would have $\lim _{i \rightarrow \infty} \operatorname{Lip}\left(f_{i}\right)=1$. The same argument would work for $f_{i}^{-1}$.

Let us now prove (3.8). In what follows we shall assume that $\rho, \rho_{i}$ have $\mathbb{S}^{n}$ as their domain of definition. As $\rho-r$ is bounded from below, again by Lemma 2.1, it is enough to prove $\lim _{i \rightarrow \infty} \operatorname{Lip}\left(\rho_{i}-\rho\right)=0$. Let us denote by $h_{i}^{*}, h^{*}$ the support functions of the polar sets $C_{i}^{*}, C^{*}$ of $C_{i}, C$, respectively. By [51, Remark 1.7.7], $h_{i}^{*}=1 / \rho_{i}$. Since $\rho_{i}$ is uniformly bounded from below, again by Lemma 2.1 it is enough to check that $\operatorname{Lip}\left(h_{i}^{*}-h^{*}\right) \rightarrow 0$. By Lemma 3.2, the convex functions $h_{i}^{*}$ converge pointwise to $h^{*}$. Lemma 3.3 then implies that $\operatorname{Lip}\left(h_{i}^{*}-h^{*}\right)=0$.

Remark 3.5. Observe that the map given by (3.6) is defined in all of $\mathbb{R}^{n+1}$ and takes $C$ onto $C_{i}$ and $\mathbb{R}^{n+1} \backslash C$ onto $\mathbb{R}^{n+1} \backslash C_{i}$.

Remark 3.6. If $f: C_{1} \rightarrow C_{2}$ is a bi-Lipschitz map between convex bodies of $\mathbb{R}^{n+1}$, then $g: \lambda C_{1} \rightarrow \lambda C_{2}$, defined by $g(x)=\lambda f\left(\frac{x}{\lambda}\right)$, is also bi-Lipschitz and satisfies $\operatorname{Lip}(f)=\operatorname{Lip}(g), \operatorname{Lip}\left(f^{-1}\right)=\operatorname{Lip}\left(g^{-1}\right)$.

Remark 3.7. Let $C, C^{\prime} \subset \mathbb{R}^{n+1}$ be two convex bodies so that $\delta\left(C, C^{\prime}\right)>0$, $d_{L}\left(C, C^{\prime}\right)>0$ (it is enough to consider two non-isometric convex bodies). For $i \in \mathbb{N}$, we have

$$
d_{L}\left(i C, i C^{\prime}\right)=d_{L}\left(i^{-1} C, i^{-1} C^{\prime}\right)=d_{L}\left(C, C^{\prime}\right) .
$$

On the other hand,

$$
\delta\left(i C, i C^{\prime}\right)=i \delta\left(C, C^{\prime}\right) \rightarrow+\infty ; \quad \delta\left(i^{-1} C, i^{-1} C^{\prime}\right)=i^{-1} \delta\left(C, C^{\prime}\right) \rightarrow 0 .
$$


Hence Lipschitz and Hausdorff distances will not be equivalent in a subset of the space of convex bodies unless we impose uniform bounds on the circumradius and the inradius.

Now we prove that the convergence of a sequence of convex bodies in Lipschitz distance, together with an upper bound on the circumradii of the elements of the sequence, implies the convergence of a subsequence in Hausdorff distance to a convex body isometric to the Lipschitz limit. We recall that it is known that Lipschitz convergence implies Gromov-Hausdorff convergence; see [27, Prop. 3.7], 13, Ex. 7.4.3].

Theorem 3.8. Let $\left\{C_{i}\right\}_{i \in \mathbb{N}}$ be a sequence of convex bodies converging to a convex body $C$ in Lipschitz distance. Then $\left\{C_{i}\right\}_{i \in \mathbb{N}}$ converges to $C$ in weak Hausdorff distance.

Proof. Let $f_{i}: C \rightarrow C_{i}$ be a sequence of bi-Lipschitz maps with $\operatorname{Lip}\left(f_{i}\right), \operatorname{Lip}\left(f_{i}^{-1}\right) \rightarrow$ 1. Then $\operatorname{diam}\left(C_{i}\right)$ are uniformly bounded, so that translating the sets $C_{i}$ we may assume they are uniformly bounded. Applying the Arzelà-Ascoli Theorem, a subsequence of $f_{i}$ uniformly converges to a lipschitz map $f: C \rightarrow \mathbb{R}^{n+1}$. We shall assume the whole sequence converges. The sequence $C_{i}=f_{i}(C)$ converges to the compact set $f(C)$ in the sense of Kuratowski [5, Def. 4.4.13] and so converges to $f(C)$ in Hausdorff distance by [5, Prop. 4.4.14]. To check that $C_{i}$ converges to $f(C)$ in the sense of Kuratowski we take $x=\lim _{k \rightarrow \infty} f_{i_{k}}\left(x_{i_{k}}\right)$, with $x_{i_{k}} \in C$, and we extract a convergent subsequence of $x_{i_{k}}$ to some $x_{0} \in C$ to get $x=f\left(x_{0}\right) \in f(C)$. On the other hand, every $x \in f(C)$ is the limit of the sequence of points $f_{i}(x) \in C_{i}$.

Since $f_{i} \rightarrow f$ and $\operatorname{Lip}\left(f_{i}\right) \rightarrow 1$, Lemma 2.1 implies $\operatorname{Lip}(f) \leqslant 1$ and $|f(x)-f(y)| \leqslant$ $|x-y|$ for any $x, y \in C$. On the other hand, taking limits when $i \rightarrow \infty$ in the inequalities

$$
|x-y|=\left|f_{i}^{-1}\left(f_{i}(x)\right)-f_{i}^{-1}\left(f_{i}(y)\right)\right| \leqslant \operatorname{Lip}\left(f_{i}^{-1}\right)\left|f_{i}(x)-f_{i}(y)\right|,
$$

we get $|x-y| \leqslant|f(x)-f(y)|$ and so $f$ is an isometry. This argument shows that any subsequence of $\left\{C_{i}\right\}_{i \in \mathbb{N}}$ has a convergent subsequence in weak Hausdorff distance to $C$, which is enough to conclude that $\lim _{i \rightarrow \infty} \delta_{S}\left(C_{i}, C\right)=0$.

In the next result we shall obtain a geometric upper bound for the Lipschitz constant of the map built in the proof of Theorem 3.4. Observe that the same bound holds for the inverse mapping, which satisfies the same geometrical condition.

Corollary 3.9. Let $C, C^{\prime} \subset \mathbb{R}^{n+1}$ be convex bodies so that $\bar{B}(0,2 r) \subset C \cap C^{\prime}$, $C \cup C^{\prime} \subset \bar{B}(0, R) \subset \mathbb{R}^{n+1}$. Let $f: C \rightarrow C^{\prime}$ be the bi-Lipschitz map defined by

$$
f(x)= \begin{cases}x, & |x| \leqslant r, \\ r \frac{x}{|x|}+(|x|-r) \frac{\rho^{\prime}\left(\frac{x}{|x|}\right)-r}{\rho\left(\frac{x}{|x|}\right)-r} \frac{x}{|x|}, & |x| \geqslant r .\end{cases}
$$

Then we have

$$
1 \leqslant \operatorname{Lip}(f), \operatorname{Lip}\left(f^{-1}\right) \leqslant 1+\frac{R}{r}\left(\frac{R}{r}-1\right)\left(\frac{R^{2}}{r^{2}}+1\right) .
$$

Proof. By Lemma 2.1 we get $\operatorname{Lip}(f) \geqslant \operatorname{Lip}\left(\left.f\right|_{\{|x| \leqslant r\}}\right)=1$, and the same argument is valid for $f^{-1}$ as well. So in what follows we assume that $|x| \geqslant r$. Observe that 
$x \in \mathbb{R}^{n+1} \backslash B(0, r) \mapsto r \frac{x}{|x|}$ is the metric projection onto the convex set $\{|x| \leqslant r\}$ and so has Lipschitz constant 1, thus

$$
\operatorname{Lip}\left(\frac{x}{|x|}\right) \leqslant 1 / r
$$

We denote by $\rho, \rho^{\prime}$ the radial functions of $C, C^{\prime}$ respectively. Let us estimate first the Lipschitz constant of the map

$$
x \in \mathbb{R}^{n+1} \backslash B(0, r) \mapsto \frac{\rho^{\prime}\left(\frac{x}{|x|}\right)-r}{\rho\left(\frac{x}{|x|}\right)-r} .
$$

By Lemma 2.1 (i), (iii), (vii), and (3.11) we get

$$
\operatorname{Lip}\left(\frac{\rho^{\prime}\left(\frac{x}{|x|}\right)-r}{\rho\left(\frac{x}{|x|}\right)-r}\right) \leqslant \frac{1}{r} \frac{R^{2}}{r} \frac{1}{r}+(R-r) \frac{R^{2}}{r} \frac{1}{r} \frac{1}{r}=\frac{R^{2}}{r^{3}}+(R-r) \frac{R^{2}}{r^{4}} .
$$

As the above function is bounded from above by $\frac{R-r}{r}$ and $x \mapsto \frac{x}{|x|}$ is bounded from above by 1 , having Lipschitz constant no larger than $1 / r$ by (3.11), Lemma 2.1 (iv) then implies

$$
\operatorname{Lip}\left(\frac{\rho^{\prime}\left(\frac{x}{|x|}\right)-r}{\rho\left(\frac{x}{|x|}\right)-r}\right) \frac{x}{|x|} \leqslant \frac{R^{2}}{r^{3}}+(R-r) \frac{R^{2}}{r^{4}}+\frac{R-r}{r} \frac{1}{r} .
$$

Thus, as the above function is bounded from above by $\frac{R-r}{r}$ and $x \mapsto|x|-r$ is bounded from above by $R-r$, having Lipschitz constant no larger than 1 , then from Lemma 2.1 (iv) we get

$$
\begin{aligned}
\operatorname{Lip}(f) & \leqslant 1+(R-r)\left(\frac{R^{2}}{r^{3}}+(R-r) \frac{R^{2}}{r^{4}}+\frac{R-r}{r^{2}}\right)+\frac{R-r}{r} \\
& \leqslant 1+\left(\frac{R-r}{r}\right)\left(\frac{R^{2}}{r^{2}}+\left(\frac{R-r}{r}\right) \frac{R^{2}}{r^{2}}+\frac{R-r}{r}+1\right) \\
& \leqslant 1+\left(\frac{R}{r}-1\right)\left(\frac{R^{3}}{r^{3}}+\frac{R}{r}\right) .
\end{aligned}
$$

\section{The isoperimetric PRofile in the SPACE of CONVEX BOdies}

Using the results of the previous section, we shall prove in this one that when a sequence of convex bodies converges in Hausdorff distance to a convex body, the normalized isoperimetric profiles defined by (2.10) and (2.11) converge uniformly to the normalized isoperimetric profiles of the limit convex body. This has some consequences: the isoperimetric profile $I_{C}$ of a convex body $C$ and its power $I_{C}^{(n+1) / n}$, even when $C$ has non-smooth boundary, are concave. This would imply that isoperimetric regions and their complements are connected and also the connectedness of the free boundaries when the boundary is of class $C^{2, \alpha}$.

Theorem 4.1. Let $\left\{C_{i}\right\}_{i \in \mathbb{N}}$ be a sequence of convex bodies in $\mathbb{R}^{n+1}$ that converges to a convex body $C \subset \mathbb{R}^{n+1}$ in Hausdorff distance. Then $J_{C_{i}}$ converges to $J_{C}$ pointwise in $[0,1]$. Consequently, also $y_{C_{i}}$ converges pointwise to $y_{C}$. 
Proof. For $\lambda \in\{0,1\}$ we have $J_{C_{i}}(\lambda)=J_{C}(\lambda)=0$. Let us fix some $\lambda \in(0,1)$. Let $\left\{E_{i}\right\}_{i \in \mathbb{N}}$ be a sequence of isoperimetric regions in $C_{i}$ with $\left|E_{i}\right|=\lambda\left|C_{i}\right|$; see Lemma 2.3. By the regularity Lemma 2.4, $P_{C}\left(E_{i}\right)=H^{n}\left(\partial E_{i} \cap \operatorname{int}\left(C_{i}\right)\right)$. By the continuity of the volume with respect to the Hausdorff distance, we have $\lim _{i \rightarrow \infty}\left|E_{i}\right|=\lambda|C|$.

Theorem 3.4 implies the existence of a sequence of bi-Lipschitz maps $f_{i}: C_{i} \rightarrow C$ so that $\lim _{i \rightarrow \infty} \operatorname{Lip}\left(f_{i}\right)=\lim _{i \rightarrow \infty} \operatorname{Lip}\left(f_{i}\right)^{-1}=1$. Lemma 2.2 yields

$$
\begin{aligned}
& \frac{1}{\operatorname{Lip}\left(f_{i}^{-1}\right)^{n+1}}\left|E_{i}\right| \leqslant\left|f_{i}\left(E_{i}\right)\right| \leqslant \operatorname{Lip}\left(f_{i}\right)^{n+1}\left|E_{i}\right|, \\
& \frac{1}{\operatorname{Lip}\left(f_{i}^{-1}\right)^{n}} P_{C_{i}}\left(E_{i}\right) \leqslant P_{C}\left(f_{i}\left(E_{i}\right)\right) \leqslant \operatorname{Lip}\left(f_{i}\right)^{n} P_{C_{i}}\left(E_{i}\right) .
\end{aligned}
$$

So $\left\{f_{i}\left(E_{i}\right)\right\}_{i \in \mathbb{N}}$ is a sequence of finite perimeter sets in $C$ with $\lim _{i \rightarrow \infty}\left|f_{i}\left(E_{i}\right)\right|=$ $\lambda|C|$ and $\liminf _{i \rightarrow \infty} P_{C_{i}}\left(E_{i}\right)=\liminf _{i \rightarrow \infty} P_{C}\left(f_{i}\left(E_{i}\right)\right)$. From Lemma 2.3 we have

$$
\begin{aligned}
J_{C}(\lambda) & \leqslant \lim _{i \rightarrow \infty} I_{C}\left(\left|f_{i}\left(E_{i}\right)\right|\right) \leqslant \liminf _{i \rightarrow \infty} P_{C}\left(f_{i}\left(E_{i}\right)\right) \\
& =\liminf _{i \rightarrow \infty} P_{C_{i}}\left(E_{i}\right)=\liminf _{i \rightarrow \infty} J_{C_{i}}(\lambda) .
\end{aligned}
$$

Let us prove now that $J_{C}(\lambda) \geqslant \lim \sup _{i \rightarrow \infty} J_{C_{i}}(\lambda)$. We shall reason by contradiction assuming that $J_{C}(\lambda)<\limsup J_{C_{i}}(\lambda)$. Passing to a subsequence we can suppose that $\left\{J_{C_{i}}(\lambda)\right\}_{i \in \mathbb{N}}$ converges, so let us assume $J_{C}(\lambda)<\lim _{i \rightarrow \infty} J_{C_{i}}(\lambda)$. Let $E \subset C$ be an isoperimetric region with $|E|=\lambda|C|$. Consider a point $p$ in the regular part of $\partial E \cap \operatorname{int}(C)$. We take a vector field in $\mathbb{R}^{n+1}$ with compact support in a small neighborhood of $p$ that does not intersect the singular set of $\partial E$. We choose the vector field so that the deformation $\left\{E_{t}\right\}_{t \in \mathbb{R}}$ induced by the associated flow strictly increases the volume in the interval $(-\varepsilon, \varepsilon)$; i.e., $t \mapsto\left|E_{t}\right|$ is strictly increasing in $(-\varepsilon, \varepsilon)$. Taking a smaller $\varepsilon$ if necessary, the first variation formulas of volume and perimeter imply the existence of a constant $M>0$ so that

$$
\mid H^{n}\left(\partial E_{t} \cap \operatorname{int}(C)\right)-H^{n}\left(\partial E \cap \operatorname{int}(C)|\leqslant M|\left|E_{t}\right|-|E| \mid\right.
$$

holds for all $t \in(-\varepsilon, \varepsilon)$. Reducing $\varepsilon$ again if necessary we may assume

$$
H^{n}(\partial E \cap \operatorname{int}(C))+M|| E_{t}|-| E||<\lim _{i \rightarrow \infty} J_{C_{i}}(\lambda)
$$

(recall we are supposing $H^{n}(\partial E \cap \operatorname{int}(C))=J_{C}(\lambda)<\lim _{i \rightarrow \infty} J_{C_{i}}(\lambda)$ ).

For every $i \in \mathbb{N}$, consider the sets $\left\{f_{i}^{-1}\left(E_{t}\right)\right\}_{t \in(-\varepsilon, \varepsilon)}$. Since

$$
\frac{1}{\operatorname{Lip}\left(f_{i}\right)^{n+1}}\left|E_{t}\right| \leqslant\left|f_{i}^{-1}\left(E_{t}\right)\right| \leqslant \operatorname{Lip}\left(f_{i}^{-1}\right)^{n+1}\left|E_{i}\right|,
$$

$\left|E_{-\varepsilon / 2}\right|<\lambda|C|,\left|E_{\varepsilon / 2}\right|>\lambda|C|$ by the monotonicity of the function $t \mapsto\left|E_{t}\right|$ in $\left(-\frac{\varepsilon}{2}, \frac{\varepsilon}{2}\right)$, the Lipschitz constants $\operatorname{Lip}\left(f_{i}\right), \operatorname{Lip}\left(f_{i}^{-1}\right)$ converge to 1 when $i \rightarrow \infty$, and $\lim _{i \rightarrow \infty}\left|C_{i}\right| /|C|=1$, there exists $i_{0} \in \mathbb{N}$ such that

$$
\left|f_{i}^{-1}\left(E_{\varepsilon / 2}\right)\right|>\lambda\left|C_{i}\right|, \quad\left|f_{i}^{-1}\left(E_{-\varepsilon / 2}\right)\right|<\lambda\left|C_{i}\right|,
$$

for all $i \geqslant i_{0}$. Since $t \mapsto\left|f_{i}^{-1}\left(E_{t}\right)\right|$ is continuous, for every $i \geqslant i_{0}$, there exists $t(i) \in\left(-\frac{\varepsilon}{2}, \frac{\varepsilon}{2}\right)$ so that $\left|f_{i}^{-1}\left(E_{t(i)}\right)\right|=\lambda\left|C_{i}\right|$, and we have

$$
\begin{aligned}
P_{C_{i}}\left(f_{i}^{-1}\left(E_{t(i)}\right)\right) & \leqslant \operatorname{Lip}\left(f_{i}^{-1}\right) P_{C}\left(E_{t(i)}\right) \\
& \leqslant \operatorname{Lip}\left(f_{i}^{-1}\right)\left(P_{C}(E)+M \| E_{t(i)}|-| E||\right) \\
& <J_{C_{i}}(\lambda)
\end{aligned}
$$


for $i$ large enough, using (4.2) and $\operatorname{Lip}\left(f_{i}^{-1}\right) \rightarrow 1$. This contradiction shows that

$$
J_{C}(\lambda) \geqslant \limsup _{i \rightarrow \infty} J_{C_{i}}(\lambda)
$$

and hence $J_{C}(\lambda)=\lim _{i \rightarrow \infty} J_{C_{i}}(\lambda)$.

Theorem 4.1 allows us to extend properties of the isoperimetric profile for convex bodies with smooth boundary to arbitrary convex bodies. The following result was first proven by E. Milman.

Corollary 4.2 ([39, Corollary 6.11]). Let $C \subset \mathbb{R}^{n+1}$ be a convex body. Then $y_{C}$ is a concave function. As a consequence, the functions $Y_{C}, I_{C}$ and $J_{C}$ are concave.

Proof. Let $\left\{C_{i}\right\}_{i \in \mathbb{N}}$ be a sequence of convex bodies with smooth boundaries that converges to $C$ in Hausdorff distance. The functions $y_{C_{i}}$ are concave by the results of Kuwert [34]; see also [8, Remark 3.3]. By Theorem 4.1, $y_{C_{i}} \rightarrow y_{C}$ pointwise in [0,1] and so $y_{C}$ is concave. Since $Y_{C}$ is the composition of $y_{C}$ with an affine function, we conclude that $Y_{C}$ is also concave. As the composition of a concave function with an increasing concave function is concave, it follows that $I_{C}=Y_{C}^{n /(n+1)}, J_{C}=y_{C}^{n /(n+1)}$ are concave as well.

Remark 4.3. The concavity of the isoperimetric profile of a Euclidean convex body with $C^{2, \alpha}$ boundary was proven by Sternberg and Zumbrum [53]; see also [8]. Kuwert later extended this result by showing the concavity of $I_{C}^{(n+1) / n}$ for convex sets with $C^{2}$ boundary.

Corollary 4.4. Let $\left\{C_{i}\right\}_{i \in \mathbb{N}}$ be a sequence of convex bodies in $\mathbb{R}^{n+1}$ that converges to a convex body $C \subset \mathbb{R}^{n+1}$ in the Hausdorff topology. Then $J_{C_{i}}$ (resp. $y_{C_{i}}$ ) converges to $J_{C}$ (resp. $\left.y_{C}\right)$ uniformly on compact subsets of $(0,1)$.

Proof. By Theorem 4.1 we have that $J_{C_{i}} \rightarrow J_{C}$ pointwise. By [49, Thm. 10.8], this convergence is uniform on compact sets of $(0,1)$.

Corollary 4.5. Let $\left\{C_{i}\right\}_{i \in \mathbb{N}}$ be a sequence of convex bodies in $\mathbb{R}^{n+1}$ that converges to a convex body $C$ in the Hausdorff topology. Let $v_{i} \in\left[0,\left|C_{i}\right|\right], v \in[0,|C|]$ so that $v_{i} \rightarrow v$. Then $I_{C_{i}}\left(v_{i}\right) \rightarrow I_{C}(v)$.

Proof. First we consider the case $v=0$. For $i$ sufficiently large, consider Euclidean geodesic balls $B_{i} \subset \operatorname{int}\left(C_{i}\right)$ of volume $v_{i}$. Letting $v_{i} \rightarrow 0$ and taking into account that $I_{C}(0)=0$, we are done. The case $v=|C|$ is handled taking the complements $C \backslash B_{i}$ of the balls.

Now assume that $0<v<|C|$. Let $w_{i}=v_{i} /\left|C_{i}\right|$ and $w=v /|C|$. Then by the continuity of the volume with respect to the Hausdorff distance [51, Thm. 1.8.16] we get $w_{i} \rightarrow w$. Take $\varepsilon>0$ such that $[w-\varepsilon, w+\varepsilon] \subset(0,1)$. For large $i$ we have

$$
\begin{aligned}
\left|J_{C_{i}}\left(w_{i}\right)-J_{C}(w)\right| & \leqslant\left|J_{C_{i}}\left(w_{i}\right)-J_{C}\left(w_{i}\right)\right|+\left|J_{C}\left(w_{i}\right)-J_{C}(w)\right| \\
& \leqslant \sup _{x \in[w-\varepsilon, w+\varepsilon]}\left|J_{C_{i}}(x)-J_{C}(x)\right|+\left|J_{C}\left(w_{i}\right)-J_{C}(w)\right| .
\end{aligned}
$$

By Corollary 4.4, $J_{C_{i}}$ converges to $J_{C}$ uniformly on $[w-\varepsilon, w+\varepsilon]$ and, as $J_{C}$ is continuous [23], we get $J_{C_{i}}\left(w_{i}\right) \rightarrow J_{C}(w)$. From the definition of $J, w_{i}$, and $w$ the proof follows.

Theorem 4.6. Let $C \subset \mathbb{R}^{n+1}$ be a convex body and $E \subset C$ an isoperimetric region. Then $E$ and $C \backslash E$ are connected. 
Proof. We shall prove that the function $I_{C}$ satisfies

$$
I_{C}\left(v_{1}+v_{2}\right)<I_{C}\left(v_{1}\right)+I_{C}\left(v_{2}\right),
$$

whenever $v_{1}, v_{2}>0$. To prove (4.3) we shall use the concavity of $Y_{C}$ shown in Corollary 4.2 and the fact that $Y_{C}(0)=0$ to obtain

$$
\frac{Y_{C}\left(v_{1}+v_{2}\right)}{v_{1}+v_{2}} \leqslant \min \left\{\frac{Y_{C}\left(v_{1}\right)}{v_{1}}, \frac{Y_{C}\left(v_{2}\right)}{v_{2}}\right\},
$$

which implies

$$
Y_{C}\left(v_{1}+v_{2}\right) \leqslant Y_{C}\left(v_{1}\right)+Y_{C}\left(v_{2}\right),
$$

as in [7, Lemma B.1.4]. Raising to the power $n /(n+1)$ we get

$$
I_{C}\left(v_{1}+v_{2}\right) \leqslant\left(I_{C}\left(v_{1}\right)^{(n+1) / n}+I_{C}\left(v_{2}\right)^{(n+1) / n}\right)^{n /(n+1)}<I_{C}\left(v_{1}\right)+I_{C}\left(v_{1}\right),
$$

where the last inequality follows from $(a+b)^{q}<a^{q}+b^{q}$, for $a, b>0, q \in(0,1)$; cf. [30, (2.12.2)]. This proves (4.3).

If $E \subset C$ were a disconnected isoperimetric region, then $E=E_{1} \cup E_{2}$, with $|E|=\left|E_{1}\right|+\left|E_{2}\right|$, and $P_{C}(E)=P_{C}\left(E_{1}\right)+P_{C}\left(E_{2}\right)$, and we should have

$$
I_{C}(v)=P_{C}(E)=P_{C}\left(E_{1}\right)+P_{C}\left(E_{2}\right) \geqslant I_{C}\left(v_{1}\right)+I_{C}\left(v_{2}\right),
$$

which is a contradiction to (4.3). If $E \subset C$ is an isoperimetric region, then $C \backslash E$ is an isoperimetric region and so connected as well.

In case the boundary of $C$ is of class $C^{2, \alpha}$, Sternberg and Zumbrun [53] obtained an expression for the second derivative of the perimeter with respect to the volume in formula (2.31) inside Theorem 2.5 of [53. Using this formula they obtained in their Theorem 2.6 that a local minimizer $E$ of perimeter (in an $L^{1}$ sense) has the property that the closure of $\partial E \cap \operatorname{int}(C)$ is either connected or consists of a union of parallel planar (totally geodesic) components meeting $\partial C$ orthogonally with that part of $C$ lying between any two such totally geodesic components consisting of a cylinder. If $E$ is an isoperimetric region so that the closure of $\partial E \cap \operatorname{int}(C)$ consists of more than one totally geodesic component, then Theorem 2.6 in $[53$ implies that either $E$ or its complement in $C$ is disconnected, a contradiction to Theorem 4.6. So we have proven

Theorem 4.7. Let $C$ be a convex body with $C^{2, \alpha}$ boundary and $E \subset C$ be an isoperimetric region. Then the closure of $\partial E \cap \operatorname{int}(C)$ is connected.

From the concavity of $I_{C}$ the following properties of the isoperimetric profile of $I_{C}$ follow. Similar properties can be found in [6], 31, 46], [50] and [43].

Proposition 4.8. Let $C \subset \mathbb{R}^{n+1}$ be a convex body. Then

(i) $I_{C}$ can be extended continuously to $[0,|C|]$ so that $I_{C}(0)=I_{C}(|C|)=0$.

(ii) $I_{C}:[0,|C|] \rightarrow \mathbb{R}^{+}$is a positive concave function, symmetric with respect to $|C| / 2$, increasing up to $|C| / 2$ and decreasing from $|C| / 2$. Left and right derivatives $\left(I_{C}\right)_{-}^{\prime}(v),\left(I_{C}\right)_{+}^{\prime}(v)$ exist for every $v \in(0,|C|)$. Moreover, $I_{C}$ is differentiable $H^{1}$-almost everywhere and we have

$$
I_{C}(v)=\int_{0}^{v}\left(I_{C}\right)_{-}^{\prime}(w) d w=\int_{0}^{v}\left(I_{C}\right)_{+}^{\prime}(w) d w=\int_{0}^{v} I_{C}^{\prime}(w) d w,
$$

for every $v \in[0,|C|]$. 
(iii) If $E \subset C$ is an isoperimetric region of volume $v \in(0,|C|)$ and $H$ is the (constant) mean curvature of the regular part of $\partial E \cap \operatorname{int}(C)$, then

$$
\left(I_{C}\right)_{+}^{\prime}(v) \leqslant H \leqslant\left(I_{C}\right)_{-}^{\prime}(v) .
$$

In particular, if $I_{C}$ is differentiable at $v$, then the mean curvature of every isoperimetric region of volume $v$ equals $I_{C}^{\prime}(v)$.

Proof. By Theorem 4.1 we have that $I_{C}$ is a symmetric, positive, concave function, increasing up to the midpoint and then decreasing. By [49, Thm. 24.1], side derivatives exist for all volumes. By [49, Thm. 25.3] differentiability almost everywhere and absolute continuity [49, Cor. 24.2.1] hold, from where the proof of (i) follows.

To prove (ii), take an isoperimetric region $E \subset C$ of volume $v$ and constant mean curvature $H$. By the regularity Lemma 2.4 we can find an open subset $U$ contained in the regular part of $\partial E$. Take a non-trivial $C^{1}$ function $u \geqslant 0$ with compact support in $U$ that produces an inward normal variation $\left\{\phi_{t}\right\}$ for $t$ small. By the first variation of volume and perimeter we get

$$
\left.\frac{d}{d t}\right|_{t=0}\left|\phi_{t}(E)\right|=-\int_{\partial E} u,\left.\quad \frac{d}{d t}\right|_{t=0} P_{C}\left(\phi_{t}(E)\right)=-\int_{\partial E} H u .
$$

So we get $\left|\phi_{t}(E)\right|<|E|$ for $t>0$ and $\left|\phi_{t}(E)\right|>|E|$ for $t<0$. As $P_{C}\left(\phi_{t}(E)\right) \leqslant$ $I_{C}\left(\left|\phi_{t}(E)\right|\right.$, we have

$$
\left(I_{C}\right)_{-}^{\prime}(v)=\lim _{\lambda \uparrow 0} \frac{I_{C}(v+\lambda)-I_{C}(v)}{\lambda} \geqslant \frac{d P_{C}\left(\phi_{t}(E)\right)}{d\left|\phi_{t}(E)\right|}=H .
$$

Similarly replacing $u$ by $-u$ we get $\lambda>0$, so we find

$$
\left(I_{C}\right)_{+}^{\prime}(v)=\lim _{\lambda \downarrow 0} \frac{I_{C}(v+\lambda)-I_{C}(v)}{\lambda} \leqslant \frac{d P_{C}\left(\phi_{t}(E)\right)}{d\left|\phi_{t}(E)\right|}=H .
$$

Finally, we shall prove in Theorem 4.11 that convex bodies with uniform quotient circumradius/inradius satisfy a uniform relative isoperimetric inequality invariant by scaling. A similar result was proven by Bokowski and Sperner [11, Satz 3] using a map different from (3.6). A consequence of Theorem 4.11 is the existence of a uniform Poincaré inequality for balls of small radii inside convex bodies that will be proven in Theorem 4.12 and used in the next section. First we prove the following lemma.

Lemma 4.9. Let $C \subset \mathbb{R}^{n+1}$ be a convex body and $0<v_{0}<|C|$. We have

$$
I_{C}(v) \geqslant \frac{I_{C}\left(v_{0}\right)}{v_{0}^{n /(n+1)}} v^{n /(n+1)},
$$

for all $0 \leqslant v \leqslant v_{0}$. As a consequence, we get

$$
I_{C}(v) \geqslant \frac{I_{C}(|C| / 2)}{(|C| / 2)^{n /(n+1)}} \min \{v,|C|-v\}^{n /(n+1)},
$$

for all $0 \leqslant v \leqslant|C|$.

Proof. Since $Y_{C}=I_{C}^{(n+1) / n}$ is concave and $Y_{C}(0)=0$ we get

$$
\frac{Y_{C}(v)}{v} \geqslant \frac{Y_{C}\left(v_{0}\right)}{v_{0}}
$$


for $0<v \leqslant v_{0}$. Raising to the power $n /(n+1)$ we obtain (4.4). If $0 \leqslant v \leqslant|C| / 2$, then (4.5) is simply (4.4). If $|C| / 2 \leqslant v \leqslant|C|$, then $0 \leqslant|C|-v \leqslant|C| / 2$, so we apply (4.4) to $|C|-v$ with $v_{0}=|C| / 2$ and take into account that $I_{C}(v)=I_{C}(|C|-v)$ to prove (4.5).

Remark 4.10. If a set $E$ is isoperimetric in $C$ of volume $|C| / 2$, then $\lambda E$ is isoperimetric in $\lambda C$ with volume $|\lambda C| / 2$ and perimeter $P_{\lambda C}(\lambda E)=\lambda^{n} P_{C}(E)$. So the constant in (4.5) satisfies

$$
M_{C}=\frac{I_{C}(|C| / 2)}{(|C| / 2)^{n /(n+1)}}=\frac{I_{\lambda C}(|\lambda C| / 2)}{(|\lambda C| / 2)^{n /(n+1)}},
$$

for any $\lambda>0$. Hence all dilated convex sets $\lambda C$, with $\lambda>0$, satisfy the same isoperimetric inequality

$$
I_{\lambda C}(v) \geqslant M_{C} \min \{v,|\lambda C|-v\}^{n /(n+1)},
$$

for $0<v<|\lambda C|$.

Theorem 4.11. Let $C \subset \mathbb{R}^{n+1}$ be a convex body, $x, y \in C, 0<r<R$, such that $\bar{B}(y, r) \subset C \subset \bar{B}(x, R)$. Then there exists a constant $M>0$, depending only on $R / r$ and $n$, such that

$$
I_{C}(v) \geqslant M \min \{v,|C|-v\}^{n /(n+1)},
$$

for all $0 \leqslant v \leqslant|C|$.

Proof. Since $\bar{B}(y, r) \subset C \subset \bar{B}(x, R)$ we can construct a bi-Lipschitz map $f: C \rightarrow$ $\bar{B}(x, R)$ as in (3.9). Take $0<v<|C|$. By Lemma 2.3, there exists an isoperimetric set $E \subset C$ of volume $v$. By Lemma 2.2 we have

$$
\begin{gathered}
I_{C}(v)=P_{C}(E) \geqslant(\operatorname{Lip} f)^{-n} P_{B(x, R)}(f(E)), \\
|\bar{B}(x, R) \backslash f(E)| \geqslant\left(\operatorname{Lip} f^{-1}\right)^{-(n+1)}(|C \backslash E|), \\
|f(E)| \geqslant \operatorname{Lip}\left(f^{-1}\right)^{-(n+1)}|E| .
\end{gathered}
$$

We know [24, Cor. 1.29] that for $f(E) \subset \bar{B}(x, R)$ we have the isoperimetric inequality

$$
P_{\bar{B}(x, R)}(f(E)) \geqslant M(n) \min \{|f(E)|,|\bar{B}(x, R)|-|f(E)|\}^{n /(n+1)},
$$

where $M(n)$ is a constant that depends only on the dimension $n$. So we get

$$
I_{C}(v) \geqslant M(n)\left((\operatorname{Lip} f)\left(\operatorname{Lip} f^{-1}\right)\right)^{-n} \min \{v,|C|-v\}^{n /(n+1)} .
$$

As $\bar{B}(x, R) \subset \bar{B}(y, 2 R)$, Corollary 3.9 provides upper bounds of $\operatorname{Lip}(f), \operatorname{Lip}\left(f^{-1}\right)$ depending only on $R / r$. This completes the proof of the proposition.

Theorem 4.12. Let $C \subset \mathbb{R}^{n+1}$ be a convex body. Given $r_{0}>0$, there exist positive constants $M, \ell_{1}$, depending only on $r_{0}$ and $C$, and a universal positive constant $\ell_{2}$ so that

$$
I_{\bar{B}_{C}(x, r)}(v) \geqslant M \min \left\{v,\left|\bar{B}_{C}(x, r)\right|-v\right\}^{n /(n+1)},
$$

for all $x \in C, 0<r \leqslant r_{0}$, and $0<v<\left|\bar{B}_{C}(x, r)\right|$. Moreover

$$
\ell_{1} r^{n+1} \leqslant\left|\bar{B}_{C}(x, r)\right| \leqslant \ell_{2} r^{n+1},
$$

for any $x \in C, 0<r \leqslant r_{0}$. 
Proof. To prove (4.7) we only need an upper estimate of the quotient of $r$ over the inradius of $\bar{B}(x, r)$ by Theorem 4.11. By the compactness of $C$ we deduce that

$$
\inf _{x \in C} \operatorname{inr}\left(\bar{B}_{C}\left(x, r_{0}\right)\right)>0 .
$$

Hence, for every $x \in C$, we always can find a point $y(x) \in \bar{B}_{C}\left(x, r_{0}\right)$ and a positive constant $\delta>0$ independent of $x$ such that

$$
\bar{B}(y(x), \delta) \subset \bar{B}_{C}\left(x, r_{0}\right) \subset \bar{B}\left(x, r_{0}\right) .
$$

Now take $0<r \leqslant r_{0}$. Let $0<\lambda \leqslant 1$ so that $r=\lambda r_{0}$, and denote by $h_{x, \lambda}$ the homothety of center $x$ and radius $\lambda$. Then we have $h_{x, \lambda}(\bar{B}(y(x), \delta)) \subset h_{x, \lambda}\left(\bar{B}_{C}\left(x, r_{0}\right)\right)$ and so

$$
\bar{B}\left(h_{x, \lambda}(y(x)), \lambda \delta\right) \subset \bar{B}_{h_{x, \lambda}(C)}\left(x, \lambda r_{0}\right) \subset \bar{B}_{C}\left(x, \lambda r_{0}\right),
$$

since $h_{x, \lambda}(C) \subset C$ as $x \in C, 0<\lambda \leqslant 1$, and $C$ is convex. Again by Theorem 4.11, a relative isoperimetric inequality is satisfied in $\bar{B}_{C}(x, r)$ with a constant $M$ that depends only on $r_{0} / \delta$.

We now prove (4.8). Since $\left|\bar{B}_{C}(x, r)\right| \leqslant|\bar{B}(x, r)|$, it is enough to take $\ell_{2}=$ $\omega_{n+1}=|\bar{B}(0,1)|$. For the remaining inequality, using the same notation as above, we have

$$
\begin{aligned}
|\bar{B}(x, r) \cap C| & =\left|\bar{B}\left(x, \lambda r_{0}\right) \cap C\right| \geqslant\left|h_{x, \lambda}\left(\bar{B}\left(x, r_{0}\right) \cap C\right)\right| \\
& =\lambda^{n+1}\left|\bar{B}\left(x, r_{0}\right) \cap C\right| \geqslant \lambda^{n+1}|\bar{B}(y(x), \delta)| \\
& =\omega_{n+1}\left(\delta / r_{0}\right)^{n+1} r^{n+1},
\end{aligned}
$$

and we take $\ell_{1}=\omega_{n+1}\left(\delta / r_{0}\right)^{n+1}$.

\section{Convergence of isoperimetric Regions}

Let $\left\{C_{i}\right\}_{i \in \mathbb{N}}$ be a sequence of convex bodies converging in Hausdorff distance to a convex body $C$, and $\left\{E_{i}\right\}_{i \in \mathbb{N}}$ a sequence of isoperimetric regions in $C_{i}$ of volumes $v_{i}$ weakly converging to some isoperimetric region $E \subset$ of volume $v=\lim _{i \rightarrow \infty} v_{i}$. The main result in this section is that $E_{i}$ converges to $E$ in Hausdorff distance and also their relative boundaries. As a byproduct, we shall also prove that there exists always in $C$ an isoperimetric region with connected boundary. It is still an open question to show that every isoperimetric region on a convex body has connected boundary.

We prove first a number of lemmata.

Lemma 5.1. Let $C$ be a convex body and $\lambda>0$. Then

$$
I_{\lambda C}\left(\lambda^{n+1} v\right)=\lambda^{n} I_{C}(v),
$$

for all $0 \leqslant v \leqslant \min \{|C|,|\lambda C|\}$.

Proof. For $v$ in the above conditions we get

$$
\begin{aligned}
I_{\lambda C}\left(\lambda^{n+1} v\right) & =\inf \left\{P_{\lambda C}(\lambda E): \lambda E \subset \lambda C,|\lambda E|=\lambda^{n+1} v\right\} \\
& =\inf \left\{\lambda^{n} P_{C}(E): E \subset C,|E|=v\right\} \\
& =\lambda^{n} I_{C}(v) .
\end{aligned}
$$


Remark 5.2. Lemma 5.1 implies

$$
Y_{\lambda C}\left(\lambda^{n+1} v\right)=\lambda^{n+1} Y_{C}(v)
$$

for any $\lambda>0$ and $0 \leqslant v \leqslant \min \{|C|,|\lambda C|\}$.

Lemma 5.3. Let $C$ be a convex body and $\lambda \geqslant 1$. Then

$$
I_{\lambda C}(v) \geqslant I_{C}(v)
$$

for all $0 \leqslant v \leqslant|C|$.

Proof. Let $Y_{\lambda C}=I_{\lambda C}^{(n+1) / n}$. We know from Corollary 4.2 that $Y_{C}$ is a concave function with $Y_{\lambda C}(0)=0$. Since $\lambda \geqslant 1$, for $v>0$ we have

$$
\frac{Y_{\lambda C}(v)}{v} \geqslant \frac{Y_{\lambda C}\left(\lambda^{n+1} v\right)}{\lambda^{n+1} v}
$$

which implies, using (5.2),

$$
\lambda^{n+1} Y_{\lambda C}(v) \geqslant Y_{\lambda C}\left(\lambda^{n+1} v\right)=\lambda^{n+1} Y_{C}(v) .
$$

This proves (5.3).

In a similar way to [35, p. 18], given a convex body $C$ and $E \subset C$, we define a function $h: C \times(0,+\infty) \rightarrow\left(0, \frac{1}{2}\right)$ by

$$
h(E, C, x, R)=\frac{\min \left\{\left|E \cap B_{C}(x, R)\right|,\left|B_{C}(x, R) \backslash E\right|\right\}}{\left|B_{C}(x, R)\right|},
$$

for $x \in C$ and $R>0$. When $E$ and $C$ are fixed, we shall simply denote

$$
h(x, R)=h(E, C, x, R) .
$$

Lemma 5.4. For any $v>0$, consider the function $f_{v}:[0, v] \rightarrow \mathbb{R}$ defined by

$$
f_{v}(s)=s^{-n /(n+1)}\left(\left(\frac{v-s}{v}\right)^{n /(n+1)}-1\right) .
$$

Then there is a constant $0<c_{2}<1$ that does not depend on $v$ so that $f_{v}(s) \geqslant$ $-(1 / 2) v^{-n /(n+1)}$ for all $0 \leqslant s \leqslant c_{2} v$.

Proof. By continuity, $f_{v}(0)=0$. Observe that $f_{v}(v)=-v^{-n /(n+1)}$ and that for $s \in[0,1]$, we have $f_{v}(s v)=f_{1}(s) v^{-n /(n+1)}$. The derivative of $f_{1}$ in the interval $(0,1)$ is given by

$$
f_{1}^{\prime}(s)=\frac{n}{n+1} \frac{(s-1)+(1-s)^{n /(n+1)}}{s-1} s^{-1-n /(n+1)},
$$

which is strictly negative and so $f_{1}$ is strictly decreasing. Hence there exists $0<c_{2}<1$ such that $f_{1}(s) \geqslant-1 / 2$ for all $s \in\left[0, c_{2}\right]$. This implies $f_{v}(s)=$ $f_{1}(s / v) v^{-n /(n+1)} \geqslant-(1 / 2) v^{-n /(n+1)}$ for all $s \in\left[0, c_{2} v\right]$.

Now we prove a key density result for isoperimetric regions. Its proof is inspired by Lemma 4.2 of the paper by Leonardi and Rigot [35. Similar results for quasiminimizing sets were previously proven by David and Semmes [19]. 
Theorem 5.5. Let $C \subset \mathbb{R}^{n+1}$ be a convex body and $E \subset C$ an isoperimetric region of volume $0<v<|C|$. Choose $\varepsilon$ so that

$$
0<\varepsilon<\min \left\{\frac{v}{\ell_{2}}, \frac{|C|-v}{\ell_{2}}, c_{2} v, c_{2}(|C|-v), \frac{I_{C}(v)^{n+1}}{\ell_{2} 8^{n+1} v^{n}}, \frac{I_{C}(v)^{n+1}}{\ell_{2} 8^{n+1}(|C|-v)^{n}}\right\},
$$

where $c_{2}$ is the constant in Lemma 5.4 .

Then, for any $x \in C$ and $R \leqslant 1$ so that $h(x, R) \leqslant \varepsilon$, we get

$$
h(x, R / 2)=0 \text {. }
$$

Moreover, in case $h(x, R)=\left|E \cap B_{C}(x, R)\right|\left|B_{C}(x, R)\right|^{-1}$, we get $\left|E \cap B_{C}(x, R / 2)\right|=$ 0 , and in case $h(x, R)=\left|B_{C}(x, R) \backslash E\right|\left|B_{C}(x, R)\right|^{-1}$, we have $\left|B_{C}(x, R / 2) \backslash E\right|=0$.

Proof. From Lemma 4.9 we get

$$
I_{C}(w) \geqslant c_{1} w^{n /(n+1)}, \quad \text { where } \quad c_{1}=v^{-n /(n+1)} I_{C}(v),
$$

for all $0 \leqslant w \leqslant v$.

Assume first that

$$
h(x, R)=\frac{\left|E \cap B_{C}(x, R)\right|}{\left|B_{C}(x, R)\right|} .
$$

Define $m(t)=\left|E \cap B_{C}(x, t)\right|, 0<t \leqslant R$. Thus $m(t)$ is a non-decreasing function. For $t \leqslant R \leqslant 1$ we get

$m(t) \leqslant m(R)=\left|E \cap B_{C}(x, R)\right|=h(x, R)\left|B_{C}(x, R)\right| \leqslant h(x, R) \ell_{2} R^{n+1} \leqslant \varepsilon \ell_{2}<v$,

by (5.6). So we obtain $(v-m(t))>0$.

By the coarea formula, when $m^{\prime}(t)$ exists, we get

$$
m^{\prime}(t)=\frac{d}{d t} \int_{0}^{t} H^{n}\left(E \cap \partial B_{C}(x, s)\right) d s=H^{n}\left(E \cap \partial B_{C}(x, t)\right),
$$

where we have denoted $\partial B_{C}(x, t)=\partial B(x, t) \cap \operatorname{int}(C)$. Define

$$
\lambda(t)=\frac{v^{1 /(n+1)}}{(v-m(t))^{1 /(n+1)}}, \quad E(t)=\lambda(t)\left(E \backslash B_{C}(x, t)\right) .
$$

Then $E(t) \subset \lambda(t) C$ and $|E(t)|=|E|=v$. By Lemma 5.3, we get $I_{\lambda(t) C} \geqslant I_{C}$ since $\lambda(t) \geqslant 1$. Combining this with [55, Cor. 5.5.3], equation (5.10), and elementary properties of the perimeter functional, we get

$$
\begin{aligned}
I_{C}(v) & \leqslant I_{\lambda(t) C}(v) \leqslant P_{\lambda(t) C}(E(t))=\lambda^{n}(t) P_{C}\left(E \backslash B_{C}(x, t)\right) \\
& \leqslant \lambda^{n}(t)\left(P_{C}(E)-P\left(E, B_{C}(x, t)\right)+H^{n}\left(E \cap \partial B_{C}(x, t)\right)\right) \\
& \leqslant \lambda^{n}(t)\left(P_{C}(E)-P_{C}\left(E \cap B_{C}(x, t)\right)+2 H^{n}\left(E \cap \partial B_{C}(x, t)\right)\right) \\
& \leqslant \lambda^{n}(t)\left(I_{C}(v)-c_{1} m(t)^{n /(n+1)}+2 m^{\prime}(t)\right),
\end{aligned}
$$

where $c_{1}$ is the constant in (5.8). Multiplying both sides by $I_{C}(v)^{-1} \lambda(t)^{-n}$ we find

$$
\lambda(t)^{-n}-1+\frac{c_{1}}{I_{C}(v)} m(t)^{n /(n+1)} \leqslant \frac{2}{I_{C}(v)} m^{\prime}(t) .
$$

Set

$$
a=\frac{2}{I_{C}(v)}, \quad b=\frac{c_{1}}{I_{C}(v)}=\frac{1}{v^{n /(n+1)}} .
$$

From the definition (5.11) of $\lambda(t)$ we get

$$
f(m(t)) \leqslant a m^{\prime}(t) \quad H^{1} \text {-a.e., }
$$


where

$$
\frac{f(s)}{s^{n /(n+1)}}=b+\frac{\left(\frac{v-s}{v}\right)^{n /(n+1)}-1}{s^{n /(n+1)}} .
$$

By Lemma 5.4, there exists a universal constant $0<c_{2}<1$, not depending on $v$, so that

$$
\frac{f(s)}{s^{n / n+1}} \geqslant b / 2 \quad \text { whenever } \quad 0<s \leqslant c_{2} v .
$$

Since $\varepsilon \leqslant c_{2} v$ by (5.6), equation (5.17) holds in the interval $[0, \varepsilon]$. If there were $t \in[R / 2, R]$ such that $m(t)=0$, then, by monotonicity of $m(t)$, we would conclude $m(R / 2)=0$ as well. So we assume $m(t)>0$ in $[R / 2, R]$. Then by (5.15) and (5.17), we get

$$
b / 2 a \leqslant \frac{m^{\prime}(t)}{m(t)^{n / n+1}}, \quad H^{1} \text {-a.e. }
$$

Integrating between $R / 2$ and $R$ we get by (5.9)

$$
\begin{aligned}
b R / 4 a & \leqslant\left(m(R)^{1 /(n+1)}-m(R / 2)^{1 /(n+1)}\right) \leqslant m(R)^{1 /(n+1)} \\
& \leqslant\left(\varepsilon \ell_{2}\right)^{1 /(n+1)} R \leqslant\left(\varepsilon \ell_{2}\right)^{1 /(n+1)} .
\end{aligned}
$$

This is a contradiction, since $\varepsilon \ell_{2}<(b / 4 a)^{n+1}=I_{C}(v)^{n+1} /\left(8^{n+1} v^{n}\right)$ by (5.6). So the proof in case $h(x, R)=\left.\left|E \cap B_{C}(x, R)\right|\left(\mid B_{C}(x, R)\right)\right|^{-1}$ is completed.

For the remaining case, when $h(x, R)=\left|B_{C}(x, R)\right|^{-1}\left|B_{C}(x, R) \backslash E\right|$, we replace $E$ by $C \backslash E$, which is also an isoperimetric region, and we are reduced to the previous case.

Remark 5.6. Case $h(x, R)=\left|B_{C}(x, R)\right|^{-1}\left|B_{C}(x, R) \backslash E\right|$ is treated in 35 in a completely different way using the monotonicity of the isoperimetric profile in Carnot groups.

We define the sets

$$
\begin{aligned}
E_{1} & =\left\{x \in C: \exists r>0 \text { such that }\left|B_{C}(x, r) \backslash E\right|=0\right\}, \\
E_{0} & =\left\{x \in C: \exists r>0 \text { such that }\left|B_{C}(x, r) \cap E\right|=0\right\}, \\
S & =\{x \in C: h(x, r)>\varepsilon \text { for all } r \leqslant 1\} .
\end{aligned}
$$

In the same way as in Theorem 4.3 of 35 ] we get

Proposition 5.7. Let $\varepsilon$ be as in Theorem [5.5. Then we have

(i) $E_{0}, E_{1}$ and $S$ form a partition of $C$.

(ii) $E_{0}$ and $E_{1}$ are open in $C$.

(iii) $E_{0}=E(0)$ and $E_{1}=E(1)$.

(iv) $S=\partial E_{0}=\partial E_{1}$, where the boundary is taken relative to $C$.

As a consequence we get the following two corollaries.

Corollary 5.8 (Lower density bound). Let $C \subset \mathbb{R}^{n+1}$ be a convex body and $E \subset C$ an isoperimetric region of volume $v$. Then there exists a constant $M>0$ depending only on $\varepsilon$, on Poincaré constant for $r \leqslant 1$, and on an Ahlfors constant $\ell_{1}$ such that

$$
P\left(E, B_{C}(x, r)\right) \geqslant M r^{n},
$$

for all $x \in \partial E_{1}$ and $r \leqslant 1$. 
Proof. If $x \in \partial E_{1}$, the choice of $\varepsilon$ and the relative isoperimetric inequality (4.7) give

$$
\begin{aligned}
P\left(E, B_{C}(x, r)\right) & \geqslant M \min \left\{\left|E \cap B_{C}(x, r)\right|,\left|B_{C}(x, r) \backslash E\right|\right\}^{n /(n+1)} \\
& =M\left(\left|B_{C}(x, r)\right| h(x, r)\right)^{n /(n+1)} \geqslant M\left(\left|B_{C}(x, r)\right| \varepsilon\right)^{n /(n+1)} \\
& \geqslant M\left(\ell_{1} \varepsilon\right)^{n /(n+1)} r^{n} .
\end{aligned}
$$

This implies the desired inequality.

Remark 5.9. If $C_{i}$ is a sequence of convex bodies converging to a convex body $C$ in Hausdorff distance, and $E_{i} \subset C_{i}$ is a sequence of isoperimetric regions converging weakly to an isoperimetric region $E \subset C$ of volume $0<v<|C|$, then a constant $M>0$ in (5.18) can be chosen independently of $i \in \mathbb{N}$. In fact, by (5.6), the constant $\varepsilon$ depends only on $\left|E_{i}\right|,\left|C_{i}\right|-\left|E_{i}\right|$, and $I_{C_{i}}\left(\left|E_{i}\right|\right)$, which are uniformly bounded since $\left|C_{i}\right| \rightarrow|C|$ and $\left|E_{i}\right| \rightarrow|E|$. By the convergence in Hausdorff distance of $C_{i}$ to $C$, both a lower Ahlfors constant $\ell_{1}$ and a Poincaré constant can be chosen uniformly for all $i \in \mathbb{N}$.

Remark 5.10. The classical monotonicity formula for rectifiable varifolds [52] can be applied in the interior of $C$ to get the lower bound (5.18) for small $r$. Assuming $C^{2}$ regularity of the boundary of $C$ (convexity is no longer needed), a monotonicity formula for varifolds with free boundary under a boundedness condition on the mean curvature was obtained by Grüter and Jost 29. This monotonicity formula implies the lower density bound (5.18).

Now we prove that isoperimetric regions also converge in Hausdorff distance to their weak limits, which are also isoperimetric regions. It is necessary to choose a representative of the isoperimetric regions in the class of finite perimeter so that Hausdorff convergence makes sense: we simply consider the closure of the set $E_{1}$ of points of density one.

Theorem 5.11. Let $\left\{C_{i}\right\}_{i \in \mathbb{N}}$ be a sequence of convex bodies that converges in Hausdorff distance to a convex body $C$. Let $E_{i} \subset C_{i}$ be a sequence of isoperimetric regions of volumes $v_{i} \rightarrow v \in(0,|C|)$. Let $f_{i}: C_{i} \rightarrow C$ be a sequence of bi-Lipschitz maps with $\operatorname{Lip}\left(f_{i}\right), \operatorname{Lip}\left(f_{i}^{-1}\right) \rightarrow 1$. Then there is an isoperimetric set $E \subset C$ such that a subsequence of $f_{i}\left(E_{i}\right)$ converges to $E$ in Hausdorff distance. Moreover, $E_{i}$ converges to $E$ in Hausdorff distance.

Proof. The sequence $\left\{f_{i}\left(E_{i}\right)\right\}_{i \in \mathbb{N}}$ has uniformly bounded perimeter, and so a subsequence, denoted in the same way, converges in $L^{1}(C)$ to a finite perimeter set $E$, which has volume $v$. The set $E$ is isoperimetric in $C$ since the sets $E_{i}$ are isoperimetric in $C_{i}$ and $I_{C_{i}}\left(v_{i}\right) \rightarrow I_{C}(v)$ by Corollary 4.5 .

By Remark [5.9, we can choose $\varepsilon>0$ so that Theorem 5.5 holds with this $\varepsilon$ for all $i \in \mathbb{N}$. Choosing a smaller $\varepsilon$ if necessary, for any $x \in C$ and $0<r \leqslant 1$, whenever $h\left(f_{i}\left(E_{i}\right), C, x, r\right) \leqslant \varepsilon$, we get $h\left(f_{i}\left(E_{i}\right), C, x, r / 2\right)=0$.

We now prove that $f_{i}\left(E_{i}\right) \rightarrow E$ in Hausdorff distance. As $\chi_{f_{i}\left(E_{i}\right)} \rightarrow \chi_{E}$ in $L^{1}(C)$, we can choose a sequence $r_{i} \rightarrow 0$ so that

$$
\left|f_{i}\left(E_{i}\right) \triangle E\right|<r_{i}^{n+2} \text {. }
$$

Now fix some $0<r<1$ and assume that, for some subsequence, there exist $x_{i} \in f_{i}\left(E_{i}\right) \backslash E_{r}$, where $E_{r}=\{x \in C: d(x, E) \leqslant r\}$. Choose $i$ large enough so that 
$r_{i}<\min \left\{\frac{\ell_{1}}{2}, r\right\}$. Then, by (5.19),

$\left|f_{i}\left(E_{i}\right) \cap B_{C}\left(x_{i}, r_{i}\right)\right| \leqslant\left|f_{i}\left(E_{i}\right) \backslash E\right| \leqslant\left|f_{i}\left(E_{i}\right) \triangle E\right|<r_{i}^{n+2}<\frac{\ell_{1} r_{i}^{n+1}}{2} \leqslant \frac{\left|B_{C}\left(x_{i}, r_{i}\right)\right|}{2}$.

So, for $i$ large enough, we get

$$
h\left(f_{i}\left(E_{i}\right), C, x_{i}, r_{i}\right)=\frac{\left|f_{i}\left(E_{i}\right) \cap B_{C}\left(x_{i}, r_{i}\right)\right|}{\left|B_{C}\left(x_{i}, r_{i}\right)\right|}<\ell_{1}^{-1} r_{i} \leqslant \varepsilon .
$$

By Theorem [5.5] we conclude that $\left|f_{i}\left(E_{i}\right) \cap B_{C}\left(x, r_{i} / 2\right)\right|=0$. The normalization condition imposed on the isoperimetric regions implies a contradiction that shows that $f_{i}\left(E_{i}\right) \subset(E)_{r}$ for $i$ large enough. In a similar way we get that $E \subset f_{i}\left(E_{i}\right)_{r}$, which proves that the Hausdorff distance between $E$ and $f_{i}\left(E_{i}\right)$ is less than an arbitrary $r>0$. So $f_{i}\left(E_{i}\right) \rightarrow E$ in Hausdorff distance.

Now we prove $\delta\left(E_{i}, E\right) \rightarrow 0$. By the triangle inequality we have

$$
\delta\left(E_{i}, E\right) \leqslant \delta\left(f_{i}\left(E_{i}\right), E\right)+\delta\left(f_{i}\left(E_{i}\right), E_{i}\right) .
$$

It only remains to show that $\delta\left(f_{i}\left(E_{i}\right), E_{i}\right) \rightarrow 0$. For $x \in E_{i}$ we have

$$
\operatorname{dist}\left(f_{i}(x), E_{i}\right) \leqslant\left|f_{i}(x)-x\right| .
$$

Assume that $r>0$ is as in definition (3.6) of $f_{i}$. Recall that $B(0,2 r) \subset C_{i} \cap C$ and that $C_{i} \cup C \subset B(0, R)$. Then by (3.7) we get $\left|f_{i}(x)-x\right|=0$ if $|x| \leqslant r$ and

$$
\left|f_{i}(x)-x\right| \leqslant \frac{(R-r)}{r}\left|\rho_{i}\left(\frac{x}{|x|}\right)-\rho\left(\frac{x}{|x|}\right)\right|
$$

if $|x| \geqslant r$. Lemma 3.2 then implies the existence of a sequence of positive real numbers $\varepsilon_{i} \rightarrow 0$ such that $\left|f_{i}(x)-x\right| \leqslant \varepsilon_{i}$ for all $x \in E_{i}$. We conclude that

$$
f_{i}\left(E_{i}\right) \subset\left(E_{i}\right)_{\varepsilon_{i}} \text {. }
$$

Writing $E_{i}=f_{i}^{-1}\left(f_{i}\left(E_{i}\right)\right)$ and reasoning as above with $f_{i}^{-1}$ instead of $f_{i}$ we obtain

$$
E_{i} \subset\left(f_{i}\left(E_{i}\right)\right)_{\varepsilon_{i}} .
$$

By the definition of the Hausdorff distance $\delta$, we get $\delta\left(f_{i}\left(E_{i}\right), E_{i}\right) \rightarrow 0$.

Recall that in Theorem 4.7 we showed that the boundaries of isoperimetric regions in convex sets with $C^{2, \alpha}$ boundary are connected. For arbitrary convex sets we have the following.

Theorem 5.12. Let $C \subset \mathbb{R}^{n+1}$ be a convex body. For every volume $0<v<|C|$ there exists an isoperimetric region in $C$ of volume $v$ with connected boundary.

We shall use the following result in the proof of Theorem 5.12 .

Theorem 5.13. Let $\left\{C_{i}\right\}_{i \in \mathbb{N}}$ be a sequence of convex bodies converging in Hausdorff distance to a convex body $C$, and let $E_{i} \subset C_{i}$ be a sequence of isoperimetric regions converging in Hausdorff distance to an isoperimetric region $E \subset C$. Then a subsequence of $\operatorname{cl}\left(\partial E_{i} \cap \operatorname{int}\left(C_{i}\right)\right)$ converges to $\operatorname{cl}(\partial E \cap \operatorname{int}(C))$ in Hausdorff distance as well.

Proof of Theorem 5.12, Let $C_{i} \subset \mathbb{R}^{n+1}$ be convex bodies with $C^{2, \alpha}$ boundary converging to $C$ in Hausdorff distance. Let $E_{i} \subset C_{i}$ be isoperimetric regions of volumes approaching $v$. By Theorem 5.11 a subsequence of the sets $E_{i}$ converges to $E$ in Hausdorff distance, where $E \subset C$ is an isoperimetric region of volume $v$. By Theorem 5.13 a subsequence of the sets $\operatorname{cl}\left(\partial E_{i} \cap \operatorname{int}\left(C_{i}\right)\right)$ converges to $\operatorname{cl}(\partial E \cap \operatorname{int}(C))$ 
in Hausdorff distance. Theorem 4.7 implies that the sets $\operatorname{cl}\left(\partial E_{i} \cap \operatorname{int}\left(C_{i}\right)\right)$ are connected. By Proposition A.1.7 in [33], $\operatorname{cl}(\partial E \cap \operatorname{int}(C))$ is connected as well.

Proof of Theorem 5.13. We shall prove that the sequence $\left\{\operatorname{cl}\left(\partial E_{i} \cap \operatorname{int}\left(C_{i}\right)\right)\right\}_{i \in \mathbb{N}}$ converges to $\operatorname{cl}(\partial E \cap \operatorname{int}(C))$ in the Kuratowski sense [5, 4.4.13].

1. If $x=\lim _{j \rightarrow \infty} x_{i_{j}}$ for some subsequence $x_{i_{j}} \in \operatorname{cl}\left(\partial E_{i_{j}} \cap \operatorname{int}\left(C_{i}\right)\right)$, then $x \in \operatorname{cl}(\partial E \cap \operatorname{int}(C))$.

2. If $x \in \operatorname{cl}(\partial E \cap \operatorname{int}(C))$, then there exists a sequence $x_{i} \in \operatorname{cl}(\partial E \cap \operatorname{int}(C))$ converging to $x$.

Assume 1 does not hold. To simplify the notation we shall assume that $x=$ $\lim _{i \rightarrow \infty} x_{i}$, with $x_{i} \in \operatorname{cl}\left(\partial E_{i} \cap \operatorname{int}\left(C_{i}\right)\right)$. If $x \notin \operatorname{cl}(\partial E \cap \operatorname{int}(C))$ we have $x \in \operatorname{int}(E) \cup$ $\operatorname{int}(C \backslash E)$. If $x \in \operatorname{int}(E)$, then there exists $r>0$ such that $|B(x, r) \cap(C \backslash E)|=0$. Since $x_{i} \rightarrow x$ and $E_{i}, C_{i}$ converge to $E, C$ in the Hausdorff sense, respectively, we conclude by [5, Proposition 4.4.14] that $\bar{B}\left(x_{i}, r\right) \cap\left(C_{i} \backslash E_{i}\right) \rightarrow \bar{B}(x, r) \cap(C \backslash E)$ in the Hausdorff sense as well. Thus by [15, Lemma III.1.1] we get

$$
\limsup _{i \rightarrow \infty}\left|B\left(x_{i}, r\right) \cap\left(C_{i} \backslash E_{i}\right)\right| \leqslant|B(x, r) \cap(C \backslash E)|=0 .
$$

Now if $\varepsilon>0$ is as in Theorem 5.5, we get $\left|B\left(x_{i}, r\right) \cap\left(C_{i} \backslash E_{i}\right)\right| \leqslant \varepsilon$ for all large $i \in \mathbb{N}$, which implies $\left|B\left(x_{i}, r / 2\right) \cap\left(C_{i} \backslash E_{i}\right)\right|=0$. This contradicts the fact that $x_{i} \in \operatorname{cl}\left(\partial E_{i} \cap \operatorname{int}\left(C_{i}\right)\right)$. Assuming $x \in C \backslash E$ and arguing similarly we would find $\left|B\left(x_{i}, r / 2\right) \cap \operatorname{int}\left(E_{i}\right)\right|=0$. Thus $x \in \operatorname{cl}(\partial E \cap \operatorname{int}(C))$.

Assume now that 2 does not hold. Then there exists $x \in \operatorname{cl}(\partial E \cap \operatorname{int}(C))$ so that no sequence in $\operatorname{cl}\left(\partial E_{i} \cap \operatorname{int}\left(C_{i}\right)\right)$ converges to $x$. We may assume that, passing to a subsequence if necessary, there exists $\eta>0$ so that $B_{C}(x, \eta)$ does not contain any point in $\operatorname{cl}\left(\partial E_{i} \cap \operatorname{int}\left(C_{i}\right)\right)$. The radius $\eta$ can be chosen less than $\varepsilon$. Reasoning as in Case 1, we conclude that either $B_{C}(x, \eta / 2) \cap E_{i}=\emptyset$ or $B_{C}(x, \eta / 2) \cap\left(C \backslash E_{i}\right)=\emptyset$.

\section{The ASYMptotic isoperimetric PRofile of A CONVEX BODY}

In this section we shall prove that isoperimetric regions of small volume inside a convex body concentrate near boundary points whose tangent cone has the smallest possible solid angle. This will be proven by rescaling the isoperimetric regions and then studying their convergence, as in Morgan and Johnson [43. We shall recall first some results on convex cones.

Let $K \subset \mathbb{R}^{n+1}$ be a closed convex cone with vertex $p$ and let $\alpha(K)=$ $H^{n}(\partial B(p, 1) \cap \operatorname{int}(K))$ be the solid angle of $K$. It is known that the geodesic balls centered at the vertex are isoperimetric regions in $K$ [37, [47] and that they are the only ones 22 for general convex cones, without any regularity assumption on the boundary. The isoperimetric profile of $K$ is given by

$$
I_{K}(v)=\alpha(K)^{1 /(n+1)}(n+1)^{n /(n+1)} v^{n /(n+1)} .
$$

Consequently the isoperimetric profile of a convex cone is completely determined by its solid angle.

We define the tangent cone $C_{p}$ of a convex body $C$ at a given boundary point $p \in \partial C$ as the closure of the set

$$
\bigcup_{\lambda>0} h_{p, \lambda}(C)
$$

where $h_{p, \lambda}$ denotes the dilation of center $p$ and factor $\lambda$. The solid angle $\alpha\left(C_{p}\right)$ of $C_{p}$ will be denoted by $\alpha(p)$. Tangent cones to convex bodies have been widely 
considered in convex geometry under the name of supporting cones [51, $§ 2.2]$ or projection cones 12 . In the following result, we prove the lower semicontinuity of the solid angle of tangent cones in convex sets.

Lemma 6.1. Let $C \subset \mathbb{R}^{n+1}$ be a convex body, $\left\{p_{i}\right\}_{i \in \mathbb{N}} \subset \partial C$ so that $p=\lim _{i \rightarrow \infty} p_{i}$. Then

$$
\alpha(p) \leqslant \liminf _{i \rightarrow \infty} \alpha\left(p_{i}\right) .
$$

In particular, this implies the existence of points in $\partial C$ whose tangent cones are minima of the solid angle function.

Proof. We may assume that $\alpha\left(p_{i}\right)$ converges to $\lim _{i n f} \operatorname{in}_{i \rightarrow \infty} \alpha\left(p_{i}\right)$ passing to a subsequence if necessary. Since the sequence $C_{p_{i}} \cap \bar{B}\left(p_{i}, 1\right)$ is bounded for the Hausdorff distance, we can extract a subsequence (denoted in the same way) converging to a convex body $C_{\infty} \subset \bar{B}(p, 1)$. It is easy to check that $C_{\infty}$ is the intersection of a closed convex cone $K_{\infty}$ of vertex $p$ with $\bar{B}(p, 1)$ and that $C_{p} \subset K_{\infty}$. By the continuity of the volume with respect to the Hausdorff distance we have

$$
\alpha(p)=\left|C_{p} \cap \bar{B}(p, 1)\right| \leqslant\left|C_{\infty}\right|=\lim _{i \rightarrow \infty}\left|C_{p_{i}} \cap \bar{B}\left(p_{i}, 1\right)\right|=\lim _{i \rightarrow \infty} \alpha\left(p_{i}\right),
$$

yielding (6.2). To prove the existence of tangent cones with the smallest solid angle, we simply take a sequence $\left\{p_{i}\right\}_{i \in \mathbb{N}}$ of points at the boundary of $C$ so that $\alpha\left(p_{i}\right)$ converges to $\inf \{\alpha(p): p \in \partial C\}$, we extract a convergent subsequence, and we apply the lower semicontinuity of the solid angle function.

The isoperimetric profiles of tangent cones which are minima of the solid angle function coincide. The common profile will be denoted by $I_{C_{\min }}$.

Proposition 6.2. Let $C \subset \mathbb{R}^{n+1}$ be a convex body. Then

$$
I_{C}(v) \leqslant I_{C_{\min }}(v)
$$

for all $0 \leqslant v \leqslant|C|$.

Remark 6.3. A closed half-space $H \subset \mathbb{R}^{n+1}$ is a convex cone with the largest possible solid angle. Hence, for any convex body $C \subset \mathbb{R}^{n+1}$, we have

$$
I_{C}(v) \leqslant I_{H}(v),
$$

for all $0 \leqslant v \leqslant|C|$.

Remark 6.4. Proposition 6.2 gives an alternative proof of the fact that $E \cap \partial C \neq \emptyset$ when $E \subset C$ is isoperimetric since, in case $E \cap \partial C$ is empty, then $E$ is a Euclidean ball.

Proof of Proposition 6.2. Fix some volume $0<v<|C|$. Let $p \in \partial C$ such that $I_{C_{p}}=I_{C_{\min }}$. Let $r>0$ such that $\left|B_{C}(p, r)\right|=v$. The closure of the set $\partial B(p, r) \cap$ $\operatorname{int}(C)$ is a geodesic sphere of the closed cone $K_{p}$ of vertex $p$ subtended by the closure of $\partial B(p, r) \cap \operatorname{int}(C)$. If $S=\partial B(p, r) \cap \operatorname{int}(C)$, then $S=\partial B(p, r) \cap \operatorname{int}\left(K_{p}\right)$ as well. By the convexity of $C, B(p, r) \cap \operatorname{int}\left(K_{p}\right) \subset B(p, r) \cap \operatorname{int}(C)$ and so $v_{0}=$ $H^{n+1}\left(B(p, r) \cap \operatorname{int}\left(K_{p}\right)\right) \leqslant v$. Since $K_{p} \subset C_{p}$, (6.1) implies $H^{n}(S) \leqslant I_{C_{\min }}\left(v_{0}\right)$. So we have

$$
I_{C}(v) \leqslant P_{C}\left(B_{C}(p, r)\right)=H^{n}(S) \leqslant I_{C_{\min }}\left(v_{0}\right) \leqslant I_{C_{\min }}(v),
$$

as $I_{C_{\min }}$ is an increasing function. This proves (6.3). 
We now prove the following result, which strongly depends on the paper by Figalli and Indrei [22].

Lemma 6.5. Let $K \subset \mathbb{R}^{n+1}$ be a closed convex cone. Consider a sequence of sets $E_{i}$ of finite perimeter in $\operatorname{int}(K)$ such that $v_{i}=\left|E_{i}\right| \rightarrow v$. Then

$$
\liminf _{i \rightarrow \infty} P_{K}\left(E_{i}\right) \geqslant I_{K}(v) .
$$

If equality holds, then there is a family of vectors $x_{i}$ such that $x_{i}+K \subset K$, and $x_{i}+E_{i}$ converges to a geodesic ball centered at 0 of volume $v$.

Proof. We assume $K=\mathbb{R}^{k} \times \tilde{K}$, where $k \in \mathbb{N} \cup\{0\}$ and $\tilde{K}$ is a closed convex cone which contains no lines so that 0 is an apex of $\tilde{K}$. Inequality (6.4) follows from $P_{K}\left(E_{i}\right) \geqslant I_{K}\left(v_{i}\right)$ and the continuity of $I_{K}$. Let $B(w)$ be the geodesic ball in $K$ centered at 0 of volume $w>0$. If equality holds in (6.4), then

$$
\mu\left(E_{i}\right)=\left(\frac{P_{K}\left(E_{i}\right)}{I_{K}\left(v_{i}\right)}-1\right) \rightarrow 0 .
$$

Define $s_{i}$ by the equality $\left|B\left(v_{i}\right)\right|=\left|s_{i} B(v)\right|$. Obviously $s_{i} \rightarrow 1$. By Theorem 1.2 in [22] there is a sequence of points $x_{i} \in \mathbb{R}^{k} \times\{0\}$ such that

$$
\left(\frac{\left|E_{i} \triangle\left(s_{i} B(v)+x_{i}\right)\right|}{\left|E_{i}\right|}\right) \leqslant C(n, B(v))\left(\sqrt{\mu\left(E_{i}\right)}+\frac{1}{i}\right) .
$$

Since $\mu\left(E_{i}\right) \rightarrow 0$ and $\left|E_{i}\right| \rightarrow v>0$, taking limsup we get $\left|E_{i} \triangle\left(s_{i} B(v)+x_{i}\right)\right| \rightarrow 0$, and so $\left|\left(E_{i}-x_{i}\right) \triangle B(v)\right| \rightarrow 0$, which proves the result.

Theorem 6.6. Let $C \subset \mathbb{R}^{n+1}$ be a convex body. Then

$$
\lim _{v \rightarrow 0} \frac{I_{C}(v)}{I_{C_{\min }}(v)}=1
$$

Moreover, a rescaling of a sequence of isoperimetric regions of volumes approaching 0 has a convergent subsequence in Hausdorff distance to a geodesic ball centered at some vertex in a tangent cone with the smallest solid angle. The same convergence result holds for their free boundaries.

Proof. To prove (6.5) we first observe that the invariance of the tangent cone by dilations implies that (6.3) is valid for every $\lambda C$ with $\lambda>0$, i.e., $I_{\lambda C} \leqslant I_{C_{\min }}$. So we get

$$
\limsup _{i \rightarrow \infty} I_{\lambda_{i} C}(v) \leqslant I_{C_{\min }}(v),
$$

for any sequence $\left\{\lambda_{i}\right\}_{i \in \mathbb{N}}$ of positive numbers such that $\lambda_{i} \rightarrow \infty$ and any $v>0$.

Consider now a sequence $\left\{E_{i}\right\}_{i \in \mathbb{N}} \subset C$ of isoperimetric regions of volumes $v_{i} \rightarrow 0$ and $p_{i} \in E_{i} \cap \partial C$. Translating the convex set and passing to a subsequence we may assume that $p_{i} \rightarrow 0 \in \partial C$. Let $\lambda_{i}=v_{i}^{-1 /(n+1)}$. Then $\lambda_{i} \rightarrow \infty$ and $\lambda_{i} E_{i}$ are isoperimetric regions in $\lambda_{i} C$ of volume 1. By Theorem 4.6, the sets $\lambda_{i} E_{i}$ are connected. We claim that

$$
\sup _{i \in \mathbb{N}} \operatorname{diam}\left(\lambda_{i} E_{i}\right)<\infty .
$$

If the claim holds, since $p_{i} \rightarrow 0$, there is a sequence $\tau_{i} \rightarrow 0$ such that $E_{i} \subset$ $C \cap \bar{B}\left(0, \tau_{i}\right)$. Let $q \in \operatorname{int}(C \cap \bar{B}(0,1))$, and consider a solid cone $K_{q}$ with vertex $q$ 
such that $0 \in \operatorname{int}\left(K_{q}\right)$ and $K_{q} \cap C_{0} \cap \partial B(0,1)=\emptyset$. Let $s>0$ so that $\bar{B}(0, s) \subset K_{q}$. Taking $r_{i}=s^{-1} \tau_{i}, i \in \mathbb{N}$, we have

$$
r_{i}^{-1} E_{i} \subset r_{i}^{-1} C \cap \bar{B}\left(0, r_{i}^{-1} \tau_{i}\right)=r_{i}^{-1} C \cap \bar{B}(0, s) \subset K_{q} .
$$

As the sequence $r_{i}^{-1} C \cap \bar{B}(0,1)$ converges in Hausdorff distance to $C_{0} \cap \bar{B}(0,1)$ we construct, using Theorem 3.4, a family of bi-Lipschitz maps $h_{i}: r_{i}^{-1} C \cap \bar{B}(0,1) \rightarrow$ $C_{0} \cap \bar{B}(0,1)$ using a Euclidean ball $B_{q}$ contained in $\operatorname{int}(B(0,1) \cap C)$. So $h_{i}$ is the identity in $B_{q}$ and it is extended linearly along the segments leaving from $q$. By construction, the maps $h_{i}$ have the additional property

$$
P_{C_{0}}\left(h_{i}\left(r_{i}^{-1} E_{i}\right)\right)=P_{C_{0} \cap \bar{B}(0,1)}\left(h_{i}\left(r_{i}^{-1} E_{i}\right)\right) .
$$

So the sequence of bi-Lipschitz maps $g_{i}: \lambda_{i} C \cap \bar{B}\left(0, \lambda_{i} r_{i}\right) \rightarrow C_{0} \cap \bar{B}\left(0, \lambda_{i} r_{i}\right)$, obtained as in $\operatorname{Remark}$ 3.6, with the properties $\operatorname{Lip}\left(h_{i}\right)=\operatorname{Lip}\left(g_{i}\right)$ and $\operatorname{Lip}\left(h_{i}\right)=\operatorname{Lip}\left(g_{i}^{-1}\right)$ satisfies

$$
P_{C_{0}}\left(g_{i}\left(\lambda_{i} E_{i}\right)\right)=P_{C_{0} \cap \bar{B}\left(0, \lambda_{i} r_{i}\right)}\left(g_{i}\left(\lambda_{i} E_{i}\right)\right) .
$$

This property and Lemma 2.2 imply

$$
\begin{aligned}
\lim _{i \rightarrow \infty}\left|g_{i}\left(\lambda_{i} E_{i}\right)\right| & =\lim _{i \rightarrow \infty}\left|\lambda_{i} E_{i}\right|, \\
\lim _{i \rightarrow \infty} P_{C_{o}}\left(g_{i}\left(\lambda_{i} E_{i}\right)\right) & =\lim _{i \rightarrow \infty} P_{\lambda_{i} C}\left(\lambda_{i} E_{i}\right) .
\end{aligned}
$$

From these equalities, the continuity of $I_{C_{0}}$, and the fact that $\lambda_{i} E_{i} \subset \lambda_{i} C$ are isoperimetric regions of volume 1, we get

$$
I_{C_{0}}(1) \leqslant \liminf _{i \rightarrow \infty} I_{\lambda_{i} C}(1) .
$$

Combining this with (6.6) and the minimal property of $C_{\min }$ we deduce

$$
\limsup _{i \rightarrow \infty} I_{\lambda_{i} C}(1) \leqslant I_{C_{\min }}(1) \leqslant I_{C_{0}}(1) \leqslant \liminf _{i \rightarrow \infty} I_{\lambda_{i} C}(1) \text {. }
$$

Thus

$$
I_{C_{0}}(1)=I_{C_{\min }}(1)=\lim _{i \rightarrow \infty} I_{\lambda_{i} C}(1) .
$$

By (6.1), we deduce that $C_{0}$ has minimum solid angle. Finally, from (6.10), (5.1), and the fact that $\lambda C_{0}=C_{0}$ we deduce

$$
1=\lim _{i \rightarrow \infty} \frac{I_{\lambda_{i} C}(1)}{I_{C_{0}}(1)}=\lim _{i \rightarrow \infty} \frac{\lambda_{i}^{n} I_{C}\left(1 / \lambda_{i}^{n+1}\right)}{\lambda_{i}^{n} I_{C_{0}}\left(1 / \lambda_{i}^{n+1}\right)}=\lim _{i \rightarrow \infty} \frac{I_{C}\left(v_{i}\right)}{I_{C_{0}}\left(v_{i}\right)} .
$$

So it remains to prove (6.7) to conclude the proof. For this it is enough to prove

$$
P_{\lambda_{i} C}\left(F_{i}, B_{\lambda_{i} C}(x, r)\right) \geqslant M r^{n},
$$

for any $0<r \leqslant 1, x \in C$, and any isoperimetric region $F_{i} \subset \lambda_{i} C$ of volume 1 . The constant $M>0$ is independent of $i$.

To prove (6.11), observe first that the constant $M$ in the relative isoperimetric inequality (4.7) is invariant by dilations and if the factor of dilation is chosen larger than 1 , then the estimate $r \leqslant r_{0}$ is uniform. The same argument can be applied to a lower Ahlfors constant $\ell_{1}$. The constant $\ell_{2}=\omega_{n+1}=|\bar{B}(0,1)|$ is universal and does not depend on the convex set.

Now we modify the proof of Theorem 5.5 to show that there exists some $\varepsilon>0$, independent of $i$, so that if $h\left(\lambda_{i} E_{i}, \lambda_{i} C, x, r\right) \leqslant \varepsilon$, then $h\left(\lambda_{i} E_{i}, \lambda_{i} C, x, r / 2\right)=0$, for $0<r \leqslant 1$. 
First we treat the case

$$
h\left(F_{i}, \lambda_{i} C, x, R\right)=\frac{\left|F_{i} \cap B_{\lambda_{i} C}(x, R)\right|}{\left|B_{\lambda_{i} C}(x, R)\right|} .
$$

By Theorem [5.5. since $I_{C}(1) \leqslant I_{\lambda_{i} C}(1)$ for all $i \in \mathbb{N}$, it is enough to take

$$
0<\varepsilon \leqslant \min \left\{\frac{1}{\ell_{2}}, c_{2}, \frac{I_{C}(1)^{n+1}}{\ell_{2} 8^{n+1}}\right\} \text {. }
$$

Now when

$$
h\left(F_{i}, \lambda_{i} C, x, R\right)=\frac{\left|B_{\lambda_{i} C}(x, R) \backslash F_{i}\right|}{\left|B_{\lambda C}(x, R)\right|},
$$

we proceed as in the proof of Case 1 of Lemma 4.2 in [35. For $\lambda_{i}$ large enough we have $1+\ell_{2}=\left|\lambda_{i} E_{i}\right|+\ell_{2}<\left|\lambda_{i} C\right| / 2$. As $I_{\lambda_{i} C}$ is increasing in the interval $\left(0,\left|\lambda_{i} C\right| / 2\right]$ the proof of Case 1 in Lemma 4.2 of [35] provides an $\varepsilon>0$ independent of $i$.

As in Remark 5.9 we conclude the existence of $M>0$ independent of $i$ so that (6.11) holds.

Now, if $\operatorname{diam}\left(\lambda_{i} E_{i}\right)$ is not uniformly bounded, (6.11) implies that $P_{\lambda_{i} C}\left(\lambda_{i} E_{i}\right)$ is unbounded. But this contradicts the fact that $P_{\lambda_{i} C}\left(\lambda_{i} E_{i}\right)=I_{\lambda_{i} C}(1) \leqslant I_{C_{\text {min }}}(1)$ for all $i$.

Finally we prove that $\lambda_{i} E_{i}$ converges to $E$ in Hausdorff distance, where $E \subset C_{0}$ is a geodesic ball of volume 1 centered at 0 . By (6.9), $\left\{g_{i}\left(\lambda_{i} E_{i}\right)\right\}_{i \in \mathbb{N}}$ is a minimizing sequence in $C_{0}$ of volume 1. By Lemma 6.5 translating the whole sequence $\left\{g_{i}\left(\lambda_{i} E_{i}\right)\right\}_{i \in \mathbb{N}}$ if necessary we may assume it is uniformly bounded and so a subsequence of $g_{i}\left(\lambda_{i} E_{i}\right) \rightarrow E$ in $L^{1}\left(C_{0}\right)$. Theorem 5.11 implies the Hausdorff convergence of the isoperimetric regions. Theorem 5.13 implies the convergence of the free boundaries.

From Theorem 6.6 we easily get

Corollary 6.7. Let $C, K \subset \mathbb{R}^{n+1}$ be convex bodies, with $I_{C_{\min }}>I_{K_{\min }}$. Then for small volumes we have $I_{C}>I_{K}$.

For polytopes we are able to exhibit the isoperimetric regions for small volumes. The same result holds for any convex set so that there is $r>0$ such that at every point $p \in \partial C$ with tangent cone of minimum solid angle we have $B(p, r) \cap C_{p}=$ $B(p, r) \cap C$.

Theorem 6.8. Let $P \subset \mathbb{R}^{n+1}$ be a convex polytope. For small volumes the isoperimetric regions in $P$ are geodesic balls centered at vertices with the smallest solid angle.

Proof. Let $\left\{E_{i}\right\}_{i \in \mathbb{N}}$ be a sequence of isoperimetric regions in $P$ with $\left|E_{i}\right| \rightarrow 0$. By Theorem 6.6. a subsequence of $E_{i}$ is close to some vertex $x$ in $P$. Since $\operatorname{diam}\left(E_{i}\right) \rightarrow$ 0 we can suppose that, for small enough volumes, the sets $E_{i}$ are also subsets of the tangent cone $P_{x}$ and they are isoperimetric regions in $P_{x}$. By 22$]$ the only isoperimetric regions in this cone are the geodesic balls centered at $x$. These geodesic balls are also subsets of $P$.

Remark 6.9. In 21 Fall considered the partitioning problem of a domain with smooth boundary in a smooth Riemannian manifold. He showed that for small enough volume the isoperimetric regions are concentrated near the maxima of the mean curvature function and that they are asymptotic to half-spheres. The techniques used in this paper are similar to the ones used by Nardulli 44] in his study 
of isoperimetric regions of small volume in compact Riemannian manifolds. See also [43, Thm. 2.2].

Proposition 6.10. Let $C \subset \mathbb{R}^{n+1}$ be a convex body and $\left\{E_{i}\right\}_{i \in \mathbb{N}}$ a sequence of isoperimetric regions with $\left|E_{i}\right| \rightarrow 0$. Assume that $0 \in \partial C$ and that $C_{0}$ is a tangent cone with the smallest solid angle. Let $\lambda_{i}>0$ be so that $\left|\lambda_{i} E_{i}\right|=1$, and let $E \subset C_{0}$ be the geodesic ball in $C_{0}$ centered at 0 of volume 1 . Then, for every $x \in \partial E \cap \operatorname{int}\left(C_{0}\right)$ so that $B(x, r) \subset \operatorname{int}\left(C_{0}\right)$, the boundary $\partial \lambda_{i} E_{i} \cap B(x, r)$ is a smooth graph with constant mean curvature for $i$ large enough.

Proof. We use Allard's Regularity Theorem for rectifiable varifolds; see [2, [52].

Assume $\left\{E_{i}\right\}_{i \in \mathbb{N}}$ is a sequence of isoperimetric regions of volumes $v_{i} \rightarrow 0$ and that $0 \in \partial C$ is an accumulation point of points in $E_{i}$. We rescale so that $\left|\lambda_{i} E_{i}\right|=1$, project to $C_{0}$ (by means of the mapping $g_{i}$ ), and rescale again to get a minimizing sequence $F_{i}$ in $C_{0}$ of volume 1 . The sequence $\left\{F_{i}\right\}_{i \in \mathbb{N}}$ converges in $L^{1}\left(C_{0}\right)$ by Lemma 6.5.

If $v_{i}=\left|E_{i}\right| \rightarrow 0$, then $\lambda_{i}=v_{i}^{-1 /(n+1)}$. Let $H_{i}$ be the constant mean curvature of the reduced boundary of $E_{i}$. Then the mean curvature of the reduced boundary of $\lambda_{i} E_{i}$ is $\frac{1}{\lambda_{i}} H_{i}=v_{i}^{1 /(n+1)} H_{i}$. Let us check that these values are uniformly bounded.

From (4.4) we get

$$
I_{C}(v) \geqslant m v^{n /(n+1)},
$$

for all $0<v<\frac{|C|}{2}$ with $m=I_{C}(|C| / 2) /(|C| / 2)^{n /(n+1)}$. We also have

$$
I_{C}^{(n+1) / n}(v) \leqslant M v
$$

for all $0<v<|C|$. Here $M$ can be chosen as a power of the isoperimetric constant of $C_{\min }$ or $\mathbb{H}^{n+1}$ since $I_{C} \leqslant I_{C_{\min }} \leqslant I_{H}$ by Proposition 6.2 and Remark 6.3. Since $Y_{C}=I_{C}^{(n+1) / n}$ is concave, given $h>0$ small enough and using (6.13) we have

$$
\frac{Y_{C}(v)-Y_{C}(v-h)}{h} \leqslant \frac{Y_{C}(v)}{v} \leqslant M .
$$

Taking limits when $h \rightarrow 0$ we get

$$
\left(Y_{C}\right)_{-}^{\prime}(v) \leqslant M
$$

for all $0<v<|C|$. By the chain rule

$$
\left(\frac{n+1}{n}\right) I_{C}^{1 / n}(v)\left(I_{C}\right)_{-}^{\prime}(v)=\left(Y_{C}\right)_{-}^{\prime}(v) \leqslant M .
$$

Since the mean curvature $H$ of any isoperimetric region of volume $v$ satisfies $H \leqslant$ $\left(I_{C}\right)_{-}^{\prime}(v)$, using (6.12) we have

$$
\left(\frac{n+1}{n}\right) m^{1 / n} v^{1 /(n+1)} H \leqslant\left(\frac{n+1}{n}\right) I_{C}^{1 / n}(v)\left(I_{C}\right)_{-}^{\prime}(v)=\left(Y_{C}\right)_{-}^{\prime}(v) \leqslant M .
$$

So the quantity $v^{1 /(n+1)} H$ is uniformly bounded for any $0<v<|C|$. This implies that the constant mean curvature of the reduced boundary of the regions $\lambda_{i} E_{i}$ is uniformly bounded.

\section{ACKNOWLEDGEMENTS}

The authors would like to thank Frank Morgan and Gian Paolo Leonardi for their helpful suggestions and comments. 


\section{REFERENCES}

[1] Stephanie Alexander and Mohammad Ghomi, The convex hull property and topology of hypersurfaces with nonnegative curvature, Adv. Math. 180 (2003), no. 1, 324-354, DOI 10.1016/S0001-8708(03)00006-9. MR2019227 (2004k:53006)

[2] William K. Allard, On the first variation of a varifold, Ann. of Math. (2) 95 (1972), 417-491. MR0307015 (46 \#6136)

[3] F. Almgren, Spherical symmetrization, Proceedings of the International Workshop on Integral Functionals in the Calculus of Variations (Trieste, 1985), Rend. Circ. Mat. Palermo (2) Suppl. 15 (1987), 11-25. MR.934771 (89h:49037)

[4] Luigi Ambrosio, Corso introduttivo alla teoria geometrica della misura ed alle superfici minime (Italian), Appunti dei Corsi Tenuti da Docenti della Scuola. [Notes of Courses Given by Teachers at the School], Scuola Normale Superiore, Pisa, 1997. MR1736268 (2000k:49001)

[5] Luigi Ambrosio and Paolo Tilli, Topics on analysis in metric spaces, Oxford Lecture Series in Mathematics and its Applications, vol. 25, Oxford University Press, Oxford, 2004. MR2039660(2004k:28001)

[6] Christophe Bavard and Pierre Pansu, Sur le volume minimal de $\mathbf{R}^{2}$ (French), Ann. Sci. École Norm. Sup. (4) 19 (1986), no. 4, 479-490. MR875084 (88b:53048)

[7] Vincent Bayle, Propriétés de concavité du profil isopérimétrique et applications, Ph.D. thesis, Institut Fourier, 2003.

[8] Vincent Bayle and César Rosales, Some isoperimetric comparison theorems for convex bodies in Riemannian manifolds, Indiana Univ. Math. J. 54 (2005), no. 5, 1371-1394, DOI 10.1512/iumj.2005.54.2575. MR2177105 (2006f:53040)

[9] Pierre Bérard and Daniel Meyer, Inégalités isopérimétriques et applications (French), Ann. Sci. École Norm. Sup. (4) 15 (1982), no. 3, 513-541. MR690651 (84h:58147)

[10] S. G. Bobkov, On isoperimetric constants for log-concave probability distributions, Geometric aspects of functional analysis, Lecture Notes in Math., vol. 1910, Springer, Berlin, 2007, pp. 81-88, DOI 10.1007/978-3-540-72053-9_4. MR2347041 (2008j:60047)

[11] Jürgen Bokowski and Emanuel Sperner Jr., Zerlegung konvexer Körper durch minimale Trennflächen (German), J. Reine Angew. Math. 311/312 (1979), 80-100, DOI 10.1515/crll.1979.311-312.80. MR549959(81b:52010)

[12] T. Bonnesen and W. Fenchel, Theory of convex bodies, BCS Associates, Moscow, ID, 1987. Translated from the German and edited by L. Boron, C. Christenson and B. Smith. MR.920366 (88j:52001)

[13] Dmitri Burago, Yuri Burago, and Sergei Ivanov, A course in metric geometry, Graduate Studies in Mathematics, vol. 33, American Mathematical Society, Providence, RI, 2001. MR.1835418 (2002e:53053)

[14] Yu. D. Burago and V. A. Zalgaller, Geometric inequalities, Translated from the Russian by A. B. Sosinskiı̆, Grundlehren der Mathematischen Wissenschaften [Fundamental Principles of Mathematical Sciences], vol. 285, Springer Series in Soviet Mathematics, Springer-Verlag, Berlin, 1988. MR936419 (89b:52020)

[15] Isaac Chavel, Isoperimetric inequalities, Differential geometric and analytic perspectives, Cambridge Tracts in Mathematics, vol. 145, Cambridge University Press, Cambridge, 2001. MR:1849187(2002h:58040)

[16] Jaigyoung Choe, Mohammad Ghomi, and Manuel Ritoré, Total positive curvature of hypersurfaces with convex boundary, J. Differential Geom. 72 (2006), no. 1, 129-147. MR2215458 (2007a:53076)

[17] Jaigyoung Choe, Mohammad Ghomi, and Manuel Ritoré, The relative isoperimetric inequality outside convex domains in $\mathbf{R}^{n}$, Calc. Var. Partial Differential Equations 29 (2007), no. 4, 421-429, DOI 10.1007/s00526-006-0027-z. MR2329803 (2008k:58042)

[18] Jaigyoung Choe and Manuel Ritoré, The relative isoperimetric inequality in Cartan-Hadamard 3-manifolds, J. Reine Angew. Math. 605 (2007), 179-191, DOI 10.1515/CRELLE.2007.031. MR2338131 (2009c:53044)

[19] Guy David and Stephen Semmes, Quasiminimal surfaces of codimension 1 and John domains, Pacific J. Math. 183 (1998), no. 2, 213-277, DOI 10.2140/pjm.1998.183.213. MR.1625982 (99i:28012)

[20] Martin Dyer and Alan Frieze, Computing the volume of convex bodies: a case where randomness provably helps, Probabilistic combinatorics and its applications (San Francisco, CA, 
1991), Proc. Sympos. Appl. Math., vol. 44, Amer. Math. Soc., Providence, RI, 1991, pp. 123169. MR 1141926 (93a:52004)

[21] Mouhamed Moustapha Fall, Area-minimizing regions with small volume in Riemannian manifolds with boundary, Pacific J. Math. 244 (2010), no. 2, 235-260, DOI 10.2140/pjm.2010.244.235. MR2587431(2011b:53070)

[22] A. Figalli and E. Indrei, A sharp stability result for the relative isoperimetric inequality inside convex cones, J. Geom. Anal. 23 (2013), no. 2, 938-969, DOI 10.1007/s12220-011-9270-4. MR3023863

[23] Sylvestre Gallot, Inégalités isopérimétriques et analytiques sur les variétés riemanniennes, On the geometry of differentiable manifolds (Rome, 1986), Astérisque 163-164 (1988), 5-6, 31-91, 281 (1989) (French, with English summary). MR999971 (90f:58173)

[24] Enrico Giusti, Minimal surfaces and functions of bounded variation, Monographs in Mathematics, vol. 80, Birkhäuser Verlag, Basel, 1984. MR775682 (87a:58041)

[25] E. Gonzalez, U. Massari, and I. Tamanini, On the regularity of boundaries of sets minimizing perimeter with a volume constraint, Indiana Univ. Math. J. 32 (1983), no. 1, 25-37, DOI 10.1512/iumj.1983.32.32003. MR684753(84d:49043)

[26] R. E. Greene and H. Wu, Lipschitz convergence of Riemannian manifolds, Pacific J. Math. 131 (1988), no. 1, 119-141. MR917868 (89g:53063)

[27] Misha Gromov, Metric structures for Riemannian and non-Riemannian spaces. Based on the 1981 French original. With appendices by M. Katz, P. Pansu and S. Semmes. Translated from the French by Sean Michael Bates. Reprint of the 2001 English edition, Modern Birkhäuser Classics, Birkhäuser Boston Inc., Boston, MA, 2007. MR2307192 (2007k:53049)

[28] Michael Grüter, Boundary regularity for solutions of a partitioning problem, Arch. Rational Mech. Anal. 97 (1987), no. 3, 261-270, DOI 10.1007/BF00250810. MR862549 (87k:49050)

[29] Michael Grüter and Jürgen Jost, Allard type regularity results for varifolds with free boundaries, Ann. Scuola Norm. Sup. Pisa Cl. Sci. (4) 13 (1986), no. 1, 129-169. MR863638 (89d:49048)

[30] G. H. Hardy, J. E. Littlewood, and G. Pólya, Inequalities, reprint of the 1952 edition, Cambridge Mathematical Library, Cambridge University Press, Cambridge, 1988. MR.944909 (89d:26016)

[31] Wu-Yi Hsiang, On soap bubbles and isoperimetric regions in noncompact symmetric spaces. I, Tohoku Math. J. (2) 44 (1992), no. 2, 151-175, DOI 10.2748/tmj/1178227334. MR1161609 (93a:53044)

[32] R. Kannan, L. Lovász, and M. Simonovits, Isoperimetric problems for convex bodies and a localization lemma, Discrete Comput. Geom. 13 (1995), no. 3-4, 541-559, DOI 10.1007/BF02574061. MR1318794 (96e:52018)

[33] Steven G. Krantz and Harold R. Parks, The geometry of domains in space, Birkhäuser Advanced Texts: Basler Lehrbücher. [Birkhäuser Advanced Texts: Basel Textbooks], Birkhäuser Boston Inc., Boston, MA, 1999. MR1730695 (2000m:28005)

[34] Ernst Kuwert, Note on the isoperimetric profile of a convex body, Geometric analysis and nonlinear partial differential equations, Springer, Berlin, 2003, pp. 195-200. MR2008339 (2004g:49065)

[35] G. P. Leonardi and S. Rigot, Isoperimetric sets on Carnot groups, Houston J. Math. 29 (2003), no. 3, 609-637 (electronic). MR2000099 (2004d:28008)

[36] Gian Paolo Leonardi and Italo Tamanini, Metric spaces of partitions, and Caccioppoli partitions, Adv. Math. Sci. Appl. 12 (2002), no. 2, 725-753. MR1943988 (2004c:28001)

[37] Pierre-Louis Lions and Filomena Pacella, Isoperimetric inequalities for convex cones, Proc. Amer. Math. Soc. 109 (1990), no. 2, 477-485, DOI 10.2307/2048011. MR.1000160(90i:52021)

[38] Umberto Massari and Italo Tamanini, Regularity properties of optimal segmentations, J. Reine Angew. Math. 420 (1991), 61-84. MR1124566 (92h:58055)

[39] Emanuel Milman, On the role of convexity in isoperimetry, spectral gap and concentration, Invent. Math. 177 (2009), no. 1, 1-43, DOI 10.1007/s00222-009-0175-9. MR2507637 (2010j:28004)

[40] Frank Morgan, In polytopes, small balls about some vertex minimize perimeter, J. Geom. Anal. 17 (2007), no. 1, 97-106, DOI 10.1007/BF02922085. MR2302876 (2007k:49090)

[41] Frank Morgan, The Levy-Gromov isoperimetric inequality in convex manifolds with boundary, J. Geom. Anal. 18 (2008), no. 4, 1053-1057, DOI 10.1007/s12220-008-9047-6. MR2438911 (2009m:53079) 
[42] Frank Morgan, Geometric measure theory, A beginner's guide, 4th ed., Elsevier/Academic Press, Amsterdam, 2009. MR2455580 (2009i:49001)

[43] Frank Morgan and David L. Johnson, Some sharp isoperimetric theorems for Riemannian manifolds, Indiana Univ. Math. J. 49 (2000), no. 3, 1017-1041, DOI 10.1512/iumj.2000.49.1929. MR1803220(2002e:53043)

[44] Stefano Nardulli, The isoperimetric profile of a smooth Riemannian manifold for small volumes, Ann. Global Anal. Geom. 36 (2009), no. 2, 111-131, DOI 10.1007/s10455-008-9152-6. MR2529468 (2011a:53110)

[45] Stefan Peters, Convergence of Riemannian manifolds, Compositio Math. 62 (1987), no. 1, 3-16. MR892147 (88i:53076)

[46] Manuel Ritoré, The isoperimetric problem in complete surfaces of nonnegative curvature, J. Geom. Anal. 11 (2001), no. 3, 509-517, DOI 10.1007/BF02922017. MR.1857855 (2002f:53109)

[47] Manuel Ritoré and César Rosales, Existence and characterization of regions minimizing perimeter under a volume constraint inside Euclidean cones, Trans. Amer. Math. Soc. 356 (2004), no. 11, 4601-4622 (electronic), DOI 10.1090/S0002-9947-04-03537-8. MR2067135 (2005g:49076)

[48] Manuel Ritoré and Carlo Sinestrari, Mean curvature flow and isoperimetric inequalities, edited by Vicente Miquel and Joan Porti, Advanced Courses in Mathematics. CRM Barcelona, Birkhäuser Verlag, Basel, 2010. MR2590630 (2011f:53155)

[49] R. Tyrrell Rockafellar, Convex analysis, Princeton Mathematical Series, No. 28, Princeton University Press, Princeton, N.J., 1970. MR0274683 (43 \#445)

[50] Antonio Ros, Isoperimetric inequalities in crystallography, J. Amer. Math. Soc. 17 (2004), no. 2, 373-388 (electronic), DOI 10.1090/S0894-0347-03-00447-8. MR2051615(2005a:53012)

[51] Rolf Schneider, Convex bodies: the Brunn-Minkowski theory, Encyclopedia of Mathematics and its Applications, vol. 44, Cambridge University Press, Cambridge, 1993. MR 1216521 (94d:52007)

[52] Leon Simon, Lectures on geometric measure theory, Proceedings of the Centre for Mathematical Analysis, Australian National University, vol. 3, Australian National University Centre for Mathematical Analysis, Canberra, 1983. MR756417 (87a:49001)

[53] Peter Sternberg and Kevin Zumbrun, On the connectivity of boundaries of sets minimizing perimeter subject to a volume constraint, Comm. Anal. Geom. 7 (1999), no. 1, 199-220. MR.1674097 (2000d:49062)

[54] Italo Tamanini, Regularity results for almost minimal oriented hypersurfaces in $\mathbb{R}^{n}$, Quaderni del Dipartimento di Matematica dell'Università di Lecce, no. 1, Università di Lecce, http://cvgmt.sns.it/paper/1807/, 1984.

[55] William P. Ziemer, Weakly differentiable functions, Sobolev spaces and functions of bounded variation, Graduate Texts in Mathematics, vol. 120, Springer-Verlag, New York, 1989. MR $1014685(91 \mathrm{e}: 46046)$

Departamento de Geometría y Topología, Universidad de Granada, E-18071 Granada, ESPAÑA

E-mail address: ritore@ugr.es

Departamento de Geometría y Topología, Universidad de Granada, E-18071 Granada, ESPAÑA

E-mail address: stratos@ugr.es 